\title{
Extended [O II] emission around IC 418 and RX Puppis
}

\author{
D. J. Monk, M. J. Barlow and R. E. S. Clegg ^ \\ Department of Physics and Astronomy, University College London, Gower St, London WC1E 6BT
}

Accepted 1989 July 17 . Received 1989 June 19

\begin{abstract}
SUMMARY
We present Anglo-Australian Telescope (AAT) long-slit optical spectra of the planetary nebula IC 418 and the region around it, as well as similar spectra of RX Puppis and the region around it. Detectable emission in the [O II] 3726, $3729 \AA$ doublet was found all the way out to angular distances of 180 arcsec from the centre of IC 418 . The density-sensitive [O II] 3726/3729 doublet intensity ratio was found to change from a mean value of 2.2 in the central regions (corresponding to a nebular electron density of $6500 \mathrm{~cm}^{-3}$ ) to ratios of 1.0 , or less, at large angular distances, corresponding to electron densities approaching the low-density limit for this doublet ratio. We find that we do not need to invoke intrinsic emission from ionized gas situated in a halo around IC 418. Instead, both the run of emission-line surface brightness and the doublet ratio, as a function of angular distance, can be well matched by a combination of (a) the predicted seeing profile for the nebula, based on the King (1971) point source seeing profile; and (b) a uniform background due to the diffuse galactic background emission predicted for this region of the sky. It is found that atmospheric scattering of the central spectrum dominates out to $50 \mathrm{arcsec}$, while diffuse galactic emission from low-density ionized gas dominates beyond 100 arcsec. Similarly, our long-slit spectra of RX Pup show that atmospheric scattering of the central emission-line spectrum, plus ambient diffuse galactic emission, contribute to the extended $\left[\mathrm{O}_{\mathrm{II}}\right]$ emission detected around it. The relative line intensities measured in our central object spectra of IC 418 and RX Pup are also analysed and discussed.
\end{abstract}

\section{INTRODUCTION}

At optical and infrared (IR) wavelengths, IC 418 is one of the brightest planetary nebulae in the sky. It is thought to be relatively young and still optically thick to ionizing radiation. Evidence for this comes from its compactness, its high nebular electron density and surface brightness, its low nebular excitation, indicative of a relatively cool central star (confirmed by the NLTE stellar analysis of Mendez et al. 1988) and, recently, the detection of circumnebular H I by Taylor \& Pottasch (1987). Taylor, Gussie \& Goss (1989) have mapped the $\mathrm{H}$ I distribution in detail at $21 \mathrm{~cm}$, finding it to extend out to angular radii as far as $75 \mathrm{arcsec}$, and they estimated a total circumnebular $\mathrm{H}$ i mass of $0.35 \pm 0.05 M_{\odot}$.

Molecular hydrogen emission has not as yet been detected from IC 418, although the only upper limits published so far (Storey 1984) were not very sensitive. After apparently positive detections of $\mathrm{CO}$ emission (Mufson, Lyon \& Marionni 1975; Knapp et al. 1982), much more sensitive observations have produced only upper limits from the

^ Present address: Royal Greenwich Observatory, Herstmonceux Castle, Hailsham, East Sussex BN27 1RP. amount of CO around IC 418 (Clavel \& Flower 1980; Knapp 1986).

From multiaperture photometry, Willner, Becklin \& Visvanathan (1972) at 2.2 and $3.4 \mu \mathrm{m}$, and Cohen \& Barlow (1974) at $10 \mu \mathrm{m}$ found some evidence that the nebular dust emission from IC 418 extended beyond the optical boundaries. Phillips et al. $(1984,1986)$ have confirmed this by means of spatial mapping at $2.2 \mu \mathrm{m}$ and $3.5 \mu \mathrm{m}$.

Baessgen, Cerrato \& Grewing (1987) found evidence for faint extended emission around a number of bright planetary nebulae, including IC 418 from which they observed faint optical line emission out to radii of 40 arcsec, significantly beyond the bright nebular radius of 6 arcsec. They interpreted this halo emission as being due to ionized material with an $r^{-2}$ density distribution. Given that IC 418 is thought to be optically thick to ionizing radiation, the presence of low-density outflowing ionized material beyond the bright nebular boundary seems somewhat surprising.

In 1986 November, while measuring the relative intensities of a number of $\mathrm{C}$ II recombination lines in the spectrum of IC 418, we noticed that the [O II] 3726, $3729 \AA$ emission lines extended considerably beyond the bright nebular boundary. In a poster paper presented at the 1987 October 
Planetary Nebula Symposium, in Mexico City (Monk, Barlow \& Clegg 1989), we interpreted our data on the extension of the Balmer and [O $\mathrm{OI}]$ lines in terms of scattering of the central nebular radiation by dust in a large neutral halo. However, we noted that out data implied that the [O II] doublet ratio in the halo might be different from that in the core, inconsistent with a purely scattering model. For this reason, we re-observed IC 418 more extensively in 1987 December. Our new data confirmed that the $\left[\mathrm{O}_{\mathrm{II}}\right]$ doublet ratio did indeed change with increasing halo radius and, as a result, the interpretation presented in this paper differs from and supersedes that given in our earlier preliminary analysis.

In order to calibrate the effects of instrumental and atmospheric scattering, it was felt desirable to observe a point source of emission lines during our 1987 December Anglo-Australian Telescope (AAT) run. For this purpose, we choose the bright symbiotic star RX Puppis, whose free-free radio emission had been found to have a halfpower diameter of $\sim 1$ arcsec by Hollis et al. (1986).

Section 2 describes the observations made of IC 418 and RX Pup. In Section 3 we present and discuss our observations of extended emission around IC 418, while Section 4 deals with a similar emission line extension around the symbiotic star RX Pup. In Section 5, the central object emission line spectra of both IC 418 and RX Pup are discussed, while Section 6 summarizes our conclusions concerning the reality of haloes reported around bright optically thick PN such as IC 418.

\section{OBSERVATIONS}

Long-slit spectra were obtained with the UCL Image Photon Counting System (IPCS) and RGO spectrograph on the 3.9-m AAT at Siding Spring Observatory during the nights of 1986 November 9 and 10 and 1987 December 31 (UT). All of the spectra were acquired using a 1200 line $\mathrm{mm}^{-1}$ grating in first order with the $25-\mathrm{cm}$ camera, giving a spectral dispersion of $33 \AA \mathrm{mm}^{-1}$. A slit width of 1.3 arcsec was used for all observations, giving a resolution of $\sim 1.5 \AA$, as determined from the FWHM of comparison arc lines. A single spatial increment corresponded to $\sim 2.2$ arcsec along the slit, so that the 50 increments that were used gave each spectrum a total spatial coverage of 110 arcsec. Table 1 represents a $\log$ of the observations. The position angle of each exposure is given in column eight of Table 1 . The centre of IC 418 was taken to be the centroid of the most intense emission in IPCS Run 28 and the angular distance (in arcsec) of each spatial increment, in each two-dimensional (2D) image, along a radial line from this centre was calculated. A schematic diagram of the slit orientations and positions for each run, relative to the object centre, is given in Fig. 1.

The 'red' spectra of IC 418 (IPCS Runs 116 and 118; see Table 1) were taken in cloudless conditions with FWHM seeing conditions of $\sim 2$ arcsec. Neutral density (ND) filters were used to reduce the pixel count rate in $\mathrm{H} \alpha$ for each exposure. This emission line was allowed to saturate in IPCS Run 116 at a rate that would not damage the IPCS but would allow for detection of fainter emission lines. The final 'red' exposure (IPCS Run 118; see Table 1) had a higher neutral density filter and no pixel saturation occurred.

The first group of 'blue' exposures of IC 418 (IPCS Runs 155, 156 and 159; see Table 1) were taken in conditions of 'patchy' cloud with seeing conditions of $\sim 1$ arcsec. There was no pixel saturation in any of these exposures (the maximum count rate in any one pixel was $0.9 \mathrm{~Hz}$ ) and after calibration they were co-added and treated as a single image for all subsequent reduction. The spectrum obtained of IC 418 from these exposures is not used for the analysis of the extended nebular emission (Section 3.1) but is discussed in Section 5.

The second set of 'blue' exposures of IC 418 (IPCS Runs 28-31; see Table 1) was taken with a slightly higher grating angle in order to place the [O II] 3726, $29 \AA$ doublet in the centre of the spectrum for optimum focusing and resolution. The exposures were made in cloudless, dark of moon, conditions with FWHM seeing conditions around 4-5 arcsec. There was no pixel saturation in any of the exposures.

The 'blue' exposures of RX Pup (IPCS Runs 32-35; see Table 1) were taken with exactly the same instrumental setup and under the same observing conditions as for the second group of IC 418 images (IPCS Runs 28-31). There was no pixel saturation in IPCS Runs 32 or 34, but Run 33 was saturated in the strong emission lines of [ $\mathrm{Ne} \mathrm{v}] 3425 \AA$, [Ne III] $3868 \AA, \mathrm{H} 7$ and $\mathrm{H} \delta$; and Run 35 was saturated in $[\mathrm{Ne}$ III $] 3868 \AA$.

Table 1. Chronological log of observations.

\begin{tabular}{|c|c|c|c|c|c|c|c|c|}
\hline Date & $\begin{array}{c}\text { IPCS } \\
\text { Run }\end{array}$ & Object & $\begin{array}{c}\text { RA } \\
(1950)\end{array}$ & $\begin{array}{c}\text { Dec } \\
(1950)\end{array}$ & $\begin{array}{l}\text { ND } \\
\text { (dex) }\end{array}$ & $\begin{array}{c}\text { Exposure } \\
\text { Time }^{-(s e c)}\end{array}$ & PA & $\lambda$ Range \\
\hline $9 / 11 / 86$ & 116 & IC 418 & 052507.4 & -124453 & 1.3 & 650 & $135^{\circ}$ & $6400-7400 \AA$ \\
\hline$"$ & 118 & $"$ & " & $"$ & 2.6 & 110 & $"$ & $"$ \\
\hline $10 / 11 / 86$ & 155 & IC 418 & $\begin{array}{lll}05 & 25 & 09.3\end{array}$ & -124416 & 1.0 & 1460 & $183^{\circ}$ & $3400-4400 \AA$ \\
\hline$"$ & 156 & $"$ & " & $"$ & 0.7 & 2000 & $"$ & $"$ \\
\hline$"$ & 159 & $"$ & $"$ & $"$ & 1.3 & 525 & $"$ & $"$ \\
\hline $31 / 12 / 87$ & 28 & IC 418 & 052506.9 & -124419 & 2.1 & 1000 & $90^{\circ}$ & $3100-4100 \AA$ \\
\hline$"$ & 29 & IC $418(W)$ & $\begin{array}{lll}05 & 25 & 00.1\end{array}$ & -124421 & - & 640 & $"$ & $"$ \\
\hline$"$ & 30 & IC $418(N)$ & 052509.3 & $\begin{array}{lll}-124407 & 07\end{array}$ & - & 1450 & $"$ & $"$ \\
\hline$"$ & 31 & IC 418 (NW) & 052506.6 & -124406 & - & 700 & $"$ & $"$ \\
\hline$"$ & 32 & RX Pup (N) & 081228.1 & $\begin{array}{lll}-4133 & 07\end{array}$ & - & 700 & $"$ & $"$ \\
\hline$"$ & 33 & RX Pup & $\begin{array}{llll}08 & 12 & 28.1\end{array}$ & -413321 & 1.3 & 230 & $"$ & $"$ \\
\hline$"$ & 34 & $"$ & $"$ & $"$ & 1.8 & 100 & $"$ & $"$ \\
\hline$"$ & 35 & $"$ & $"$ & $"$ & 1.6 & 500 & $"$ & $"$ \\
\hline
\end{tabular}




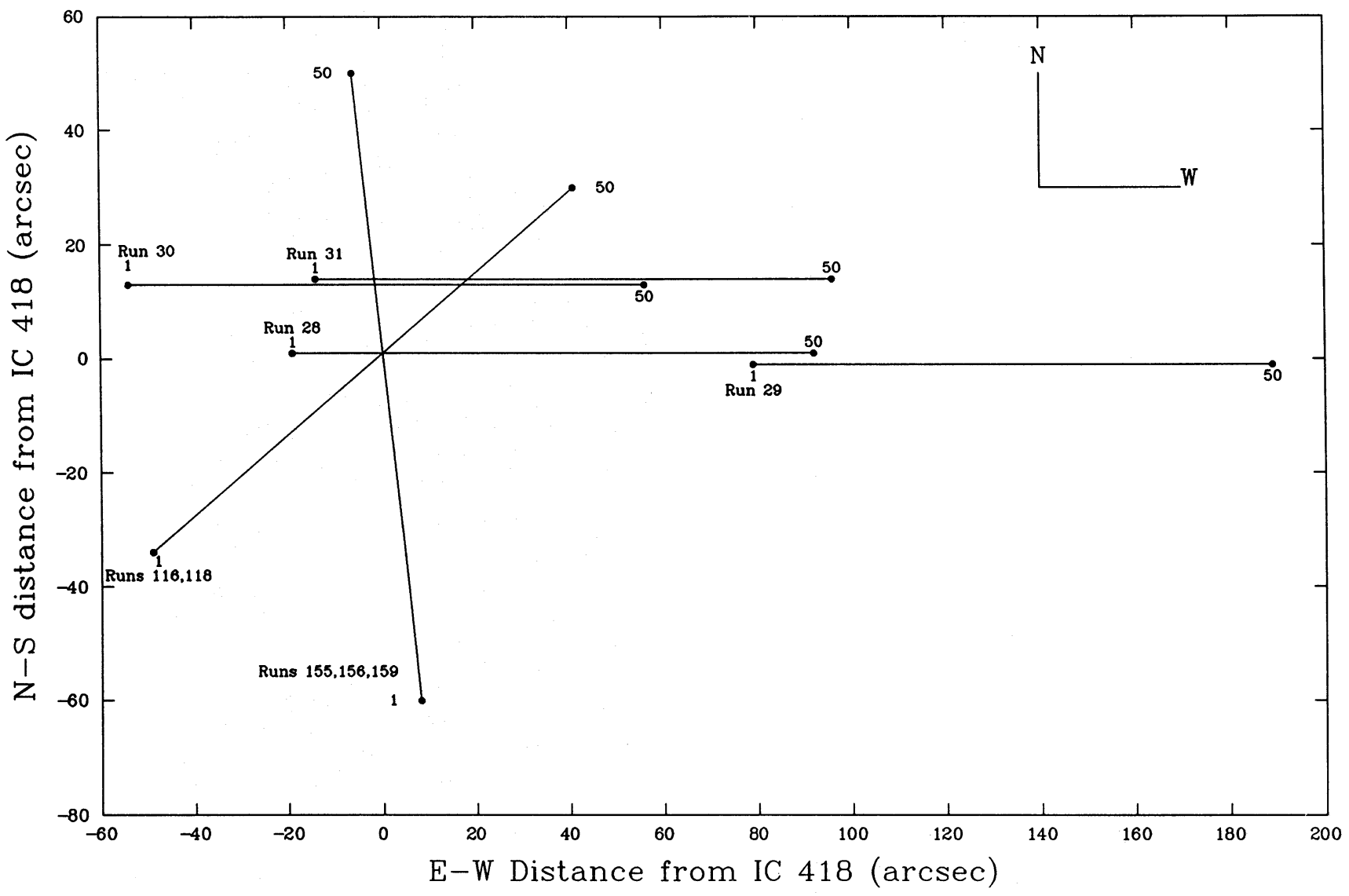

Figure 1. A schematic diagram of the slit positions and orientations relative to the centre of IC 418 , which is at 0,0 . The numbers $1-50$ at the end of each slit refer to the spatial increment numbers.

The spectra were calibrated in wavelength with respect to comparison exposures of a $\mathrm{Cu}-\mathrm{Ar}$ arc, and were calibrated spectrophotometrically by observations of the white dwarf stars L745-46A (for Runs 155, 156 and 159) and Feige 24 (for all other exposures) from the list of Oke (1974), using the FIGARO reduction package (Shortridge 1986) at the UCL STARLINK node. As some spectral lines were extended over all spatial increments of the images, the reduction did not include any subtraction of the background night sky continuum. The subsequent line intensity analysis of the calibrated spectra was carried out using the DIPSo package of routines (Howarth \& Murray 1987).

\section{EXTENDED LINE EMISSION AROUND THE PLANETARY NEBULA IC 418}

\subsection{Extended [O II] emission around IC 418}

Table 2 presents the observed [O II] $3726+3729 \AA$ surface brightness measured from each of the spectra of IC 418, as a function of angular distance from the centre of the nebula. The first column of each table gives the spatial increments in which the fluxes were measured, or the range of spatial increments that were co-added to improve the signal-to-noise of the emission lines of interest (in these cases the mean increment was used to give the mean radial distance). The second column of Table 2 gives the angular distance from the centre of the object in arcsec, the third column the [O II]
$3726+3729 \AA$ surface brightness, and the fifth column the [O II] 3726:3729 doublet intensity ratio.

The deconvolution of the partially blended [ $\left.\mathrm{O}_{\mathrm{II}}\right]$ doublet was achieved within DIPSO using the emission-line fitting routine ELF, written by Dr P. J. Storey, which determined the best fit Gaussian profiles to the observed lines, and provided formal $1 \sigma$ errors for the fit. The total formal error on the [O II] $3726: 3729$ ratio generated from these line errors is given in column six of Table 2. Error estimates are also given in these tables for the measured surface brightness of [O II] 3726:3729 $\AA$ (column four) - these errors are mainly determined by the uncertainty in the continuum level.

\subsection{Extended $\mathrm{H} \alpha$ and [N $\mathrm{II}]$ emission around IC 418}

The 'red' exposures of IC 418 (IPCS Runs 116 and 118; see Table 1) were originally intended for the detection of $\mathrm{C}$ II $6622.05 \AA$ but were also used to study the extension of $\mathrm{H} \alpha$ and [ $\left.\mathrm{N}_{\text {II }}\right] 6548,6584 \AA$ around the bright nebula. Run 118 had no saturated pixels and was used to determine the intensity of these strong emission lines in the bright nebular region, whilst Run 116 was used to measure the extended emission in these lines. There was partial blending of $\mathrm{H} \alpha$ with the $\left[\mathrm{N}_{\mathrm{II}}\right] 6548,6584 \AA$ lines and the flux in each was determined by a three-line Gaussian fit using the ELF routine. Table 3 presents the observed surface brightness of $\mathrm{H} \alpha$ and $[\mathrm{N}$ II $] 6584 \AA$ measured from these spectra, along with the $1 \sigma$ 
Table 2. Observed surface brightness of $\left[\mathrm{O}_{\mathrm{II}}\right] 3726+3729 \AA$ and the [O II] 3726:3729 doublet ratio, for IC 418 from IPCS runs 28-31.

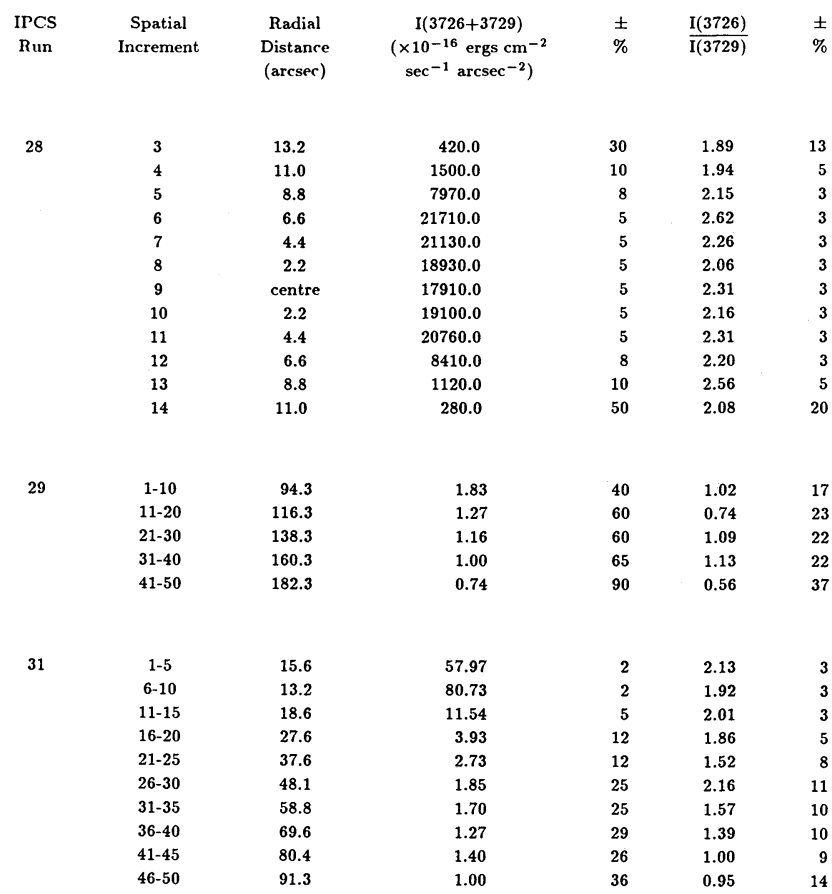

formal errors generated by the line-fitting routine, as a function of angular distance from the centre of the object.

\subsection{Seeing profiles for IC 418}

The seeing profile of a point source is discussed by King (1971), who presents a composite fit to photometric data from various sources normalized to represent the surface brightness (magnitudes per square arcsec) of a zero magnitude star as a function of angular distance from the star. The profile has a Gaussian shape out to $\sim 10$ arcsec and then follows an $R^{-2}$ slope. We reproduce this point source seeing profile in Fig. 2 (solid line) normalized to unit intensity, taken from King's fig. 1.

This point source seeing profile is, however, not suitable for extended sources such as nebulae and it is necessary to convolve the point source profile with the image of the extended object to produce a correct seeing profile. To do this, the isophotal contour maps of IC 418 in [O II] 3726, $29 \AA$ and $\mathrm{H} \alpha$, presented by Louise et al. 1987, were digitized and convolved with the point source seeing profile. The maps were normalized to the observed integrated fluxes, in cgs units, $\log F\left(\mathrm{O}_{\mathrm{II}}\right)=-9.40$ and $\log F(\mathrm{H} \alpha)=-8.96$ (Torres-Peimbert \& Peimbert 1977).

The predicted [O II] $3727 \AA$ extended source seeing profile for IC 418 (dashed line) is compared with the point source seeing profile for the same integrated flux (solid line) in Fig. 2. The two profiles are clearly different, with the extended-source profile showing a broadly flat but slightly dipped central region, an outer, brighter, edge, and then a steep fall in intensity to meet the $R^{-2}$ aureole of the point source seeing profile at larger radial distances.

The predicted $[\mathrm{O} \mathrm{II}]$ extended-source surface brightness profile for IC 418, normalized to the observed integrated flux, is presented in Fig. 3, along with the observed [O II] $3726+3739 \AA$ surface brightness distribution measured from Runs 28-31 (see Table 2). It can be seen that there is good agreement between the modelled extended source seeing profile and the observed flux out to at least 30 arcsec. Further out than this the observed surface brightness is increasingly in excess of that which could be expected from the seeing aureole alone.

Fig. 4 presents the predicted extended source seeing profile for IC 418 in $\mathrm{H} \alpha$, normalized to the observed integrated $\mathrm{H} \alpha$ flux. The shape of this profile is slightly different to that of the $[\mathrm{O} \mathrm{II}]$ profile (Fig. 3), in that it shows a slight bulge in the profile at $\sim 10$ arcsec. This is probably due to the low intensity extension of $\mathrm{H} \alpha$ out to $\sim 24$ arcsec, seen in the isophotal map of Louise et al. (1987), which itself could be a detection of the extended seeing profile and would therefore cause second-order effects to our predicted surface brightness at the edge of the bright nebula. The observed $\mathrm{H} \alpha$ surface brightness distributions from Runs 116 and 118 (see Table 3) are also shown in Fig. 4 and there is again good agreement out to at least 30 arcsec between the observed surface brightness and that predicted as due to seeing effects.

Variations in the point source seeing profile with seeing conditions (Diego 1985), or with wavelength, have not been accounted for, and the convolution of the seeing profile with the image is limited by the accuracy of the isophotal maps. However, these effects are not expected to alter the basic shape of the extended profile greatly. 
Table 3. Observed surface brightness of $\mathrm{H} \alpha$ and $[\mathrm{N}$ II] $6584 \AA$ for IC 418 from IPCS Runs 118 (central nebula) and 116 (outer extension).

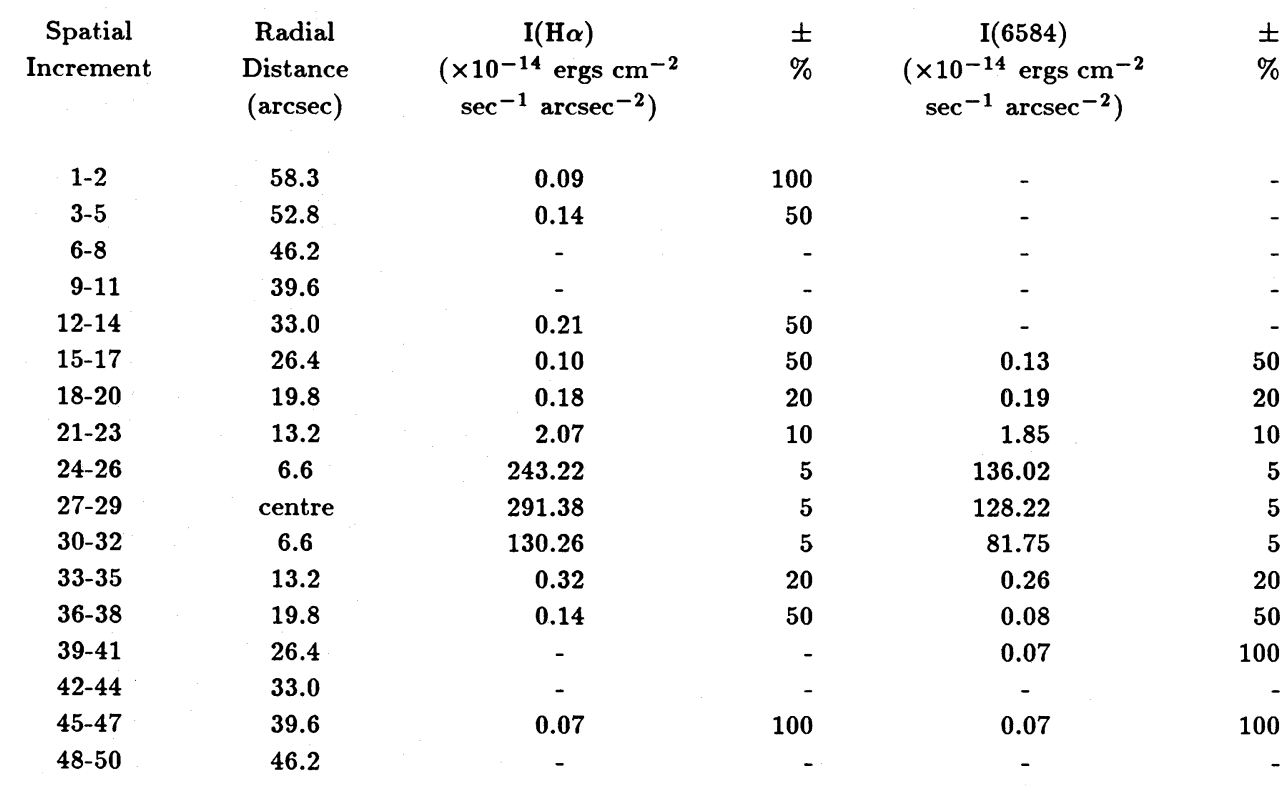

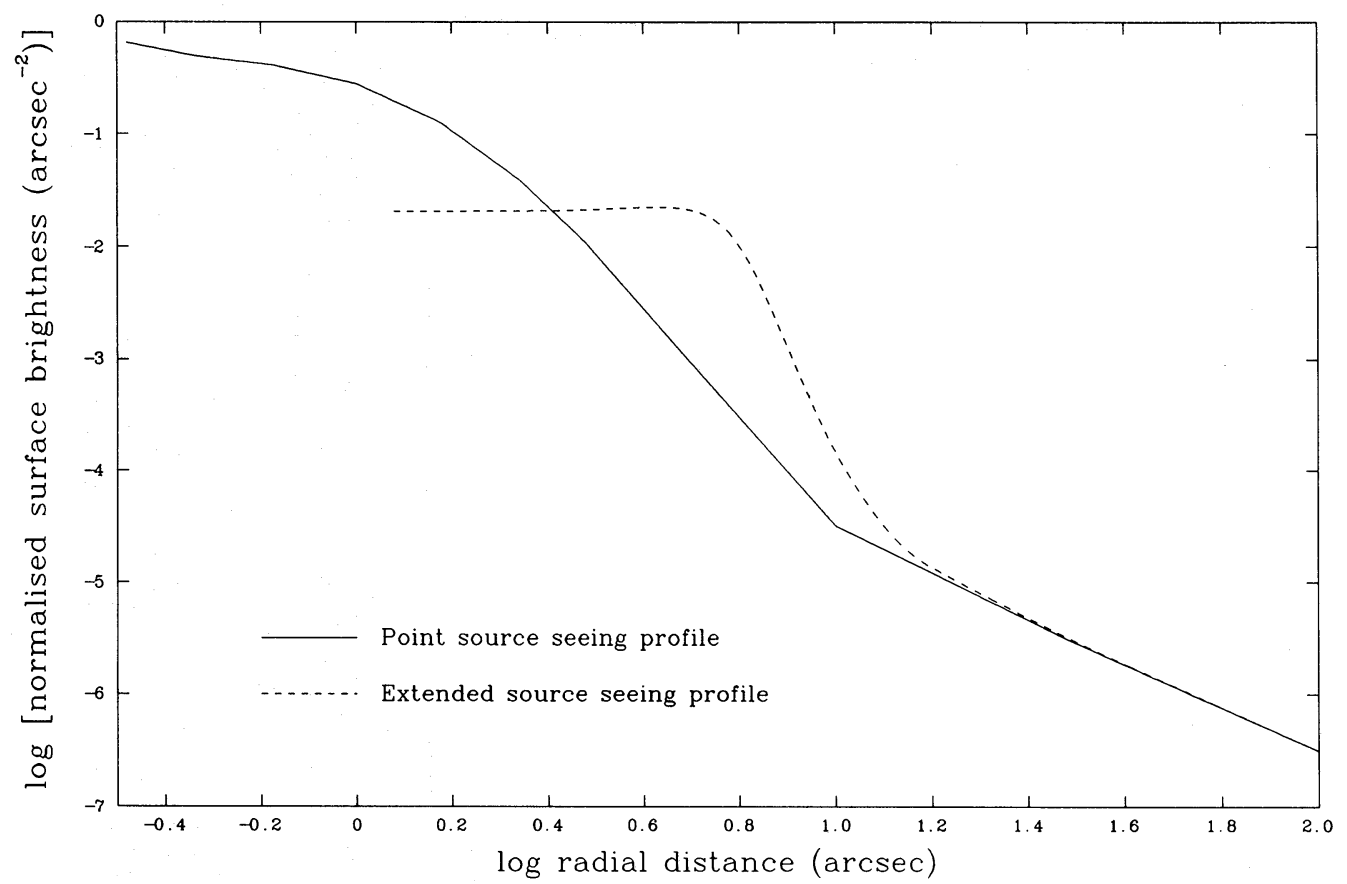

Figure 2. The solid line shows the seeing profile for a point source, normalized to unit intensity, from King (1971). The dashed line is the predicted extended source seeing profile for IC 418 . The curves are normalized to show the surface brightness divided by the total flux from the source, so that the area integral is unity.

\subsection{The [O II] doublet ratios around IC 418}

The $3726,29 \AA$ density-sensitive doublet of $[\mathrm{O}$ II $]$ was almost fully resolved by the $1200 \mathrm{~V}$ grating (see Fig. 8), and the two lines were fully deconvolved using the Gaussian fitting routine ELF. The $[\mathrm{O} \mathrm{II}]$ ratio was either measured for separate spatial increments of each spectrum, or from the mean signal obtained by co-adding contiguous increments (as indicated by column 1 of Table 2). A comparison between the $\left[\mathrm{O}_{\mathrm{II}}\right]$ ratios at the largest radial distances (Table 2, Run 29 ), and those at the centre of the object (Table 2, Run 28), indicates that an electron density difference exists between the two regions, with the outer low-density region approaching the low-density limit $[I(3726) / I(3729) \sim 0.67]$.

Fig. 5 presents the variation in the $\left[\mathrm{O}_{\mathrm{II}}\right]$ 3726:3729 ratio as a function of radial distance, based on the data presented in Table 2. As in the direct $\left[\mathrm{O}_{\mathrm{II}}\right]$ intensity image of Louise et al. (1987), we find a pronounced ring-like peaking of the

\section{Royal Astronomical Society - Provided by the NASA Astrophysics Data System}




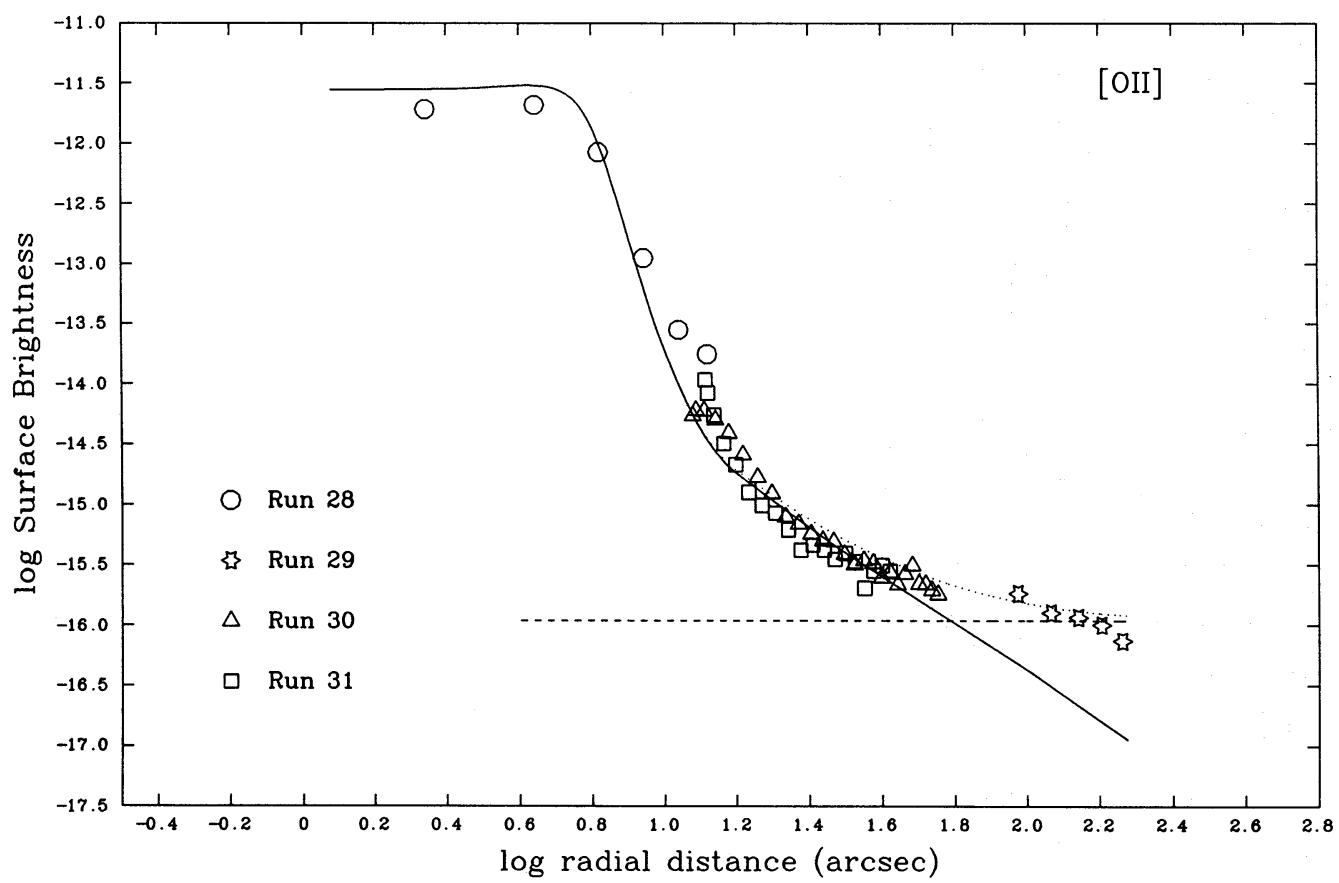

Figure 3. The solid line shows the predicted extended source surface brightness seeing profile for IC 418 in the [O II] $3726,3729 \AA$ lines and the open symbols show the measured surface brightness (in units of erg cm $\mathrm{cm}^{-2} \mathrm{~s}^{-1}$ arcsec ${ }^{-2}$ ), as a function of distance from the centre of IC 418 . The dashed line shows the predicted [ $\left.\mathrm{O}_{\mathrm{II}}\right]$ surface brightness due to diffuse galactic emission(see text), while the dotted line represents the combined level of the nebular seeing profile and diffuse galactic emission.

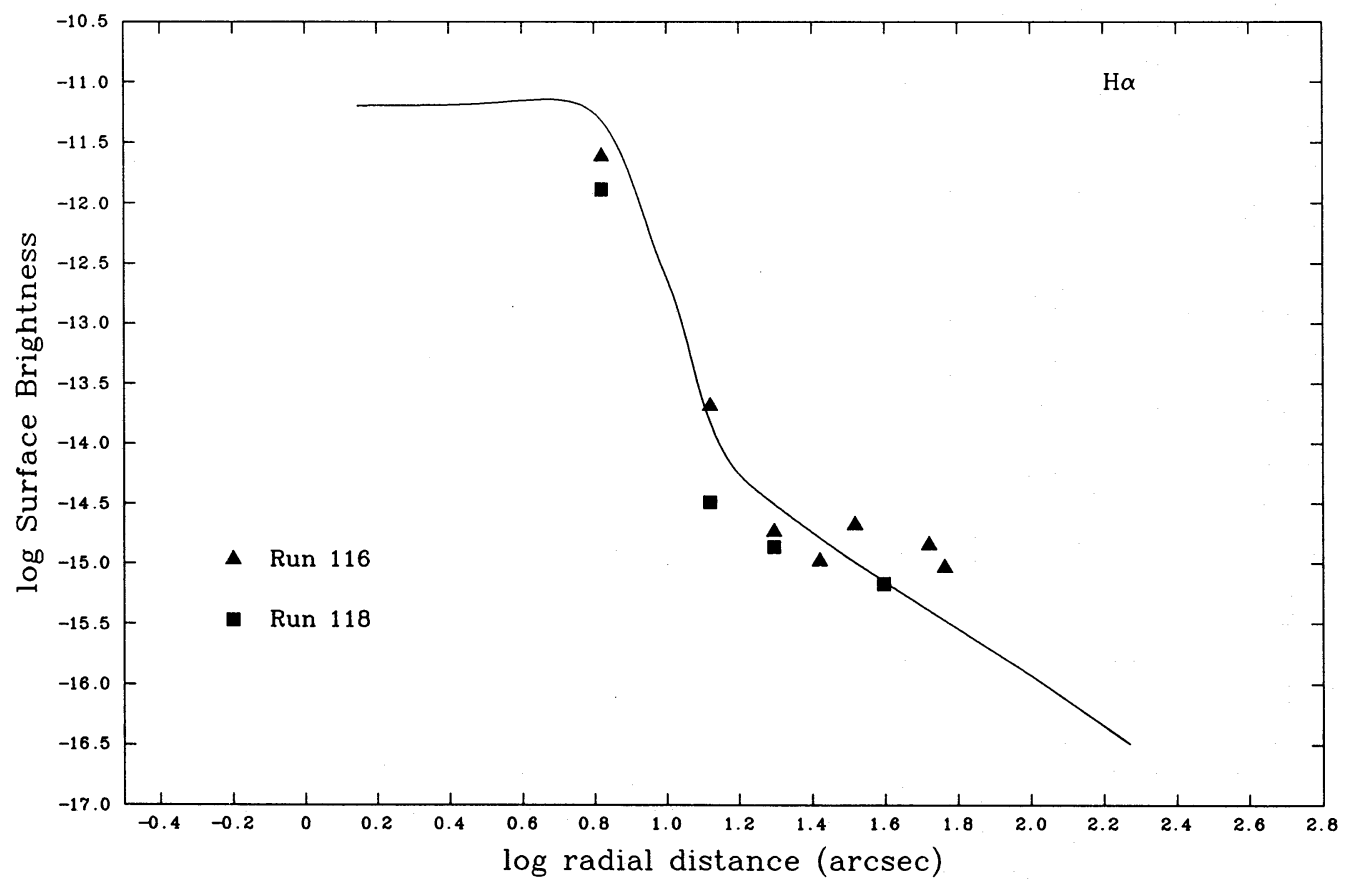

Figure 4. The solid line shows the predicted extended source surface brightness seeing profile for IC 418 in $\mathrm{H} \alpha$ and the solid symbols show the measured surface brightness (in units of erg cm${ }^{-2} \mathrm{~s}^{-1} \operatorname{arcsec}^{-2}$ ), as a function of distance from the centre of IC 418.

[O $\mathrm{II}]$ doublet ratio at the edge of the bright nebula. From Run 28 (Table 2), one finds doublet ratios as low as 2.1 near the centre of IC 418 (corresponding to an electron density of $n_{\mathrm{e}} \sim 5 \times 10^{3} \mathrm{~cm}^{-3}$ for an electron temperature of $8700 \mathrm{~K}$; see Section 5.2), rising to a peak of 2.6 at the rim intensity peak (corresponding to $n_{\mathrm{c}}=1.9 \times 10^{4} \mathrm{~cm}^{-3}$ ). Co-addition of all the spatial increments in Run 28 yields a mean [O $\mathrm{II}$ ] doublet ratio across the entire nebula of $2.25 \pm 0.02$, corresponding to $n_{\mathrm{e}}=6900 \mathrm{~cm}^{-3}$.

Fig. 5 shows that beyond the nebular rim intensity peak the $\left[\mathrm{O}_{\mathrm{II}}\right]$ doublet ratio falls steadily, reaching values near to the low-density limit at angular distances larger than $\sim 100$

\section{Royal Astronomical Society - Provided by the NASA Astrophysics Data System}




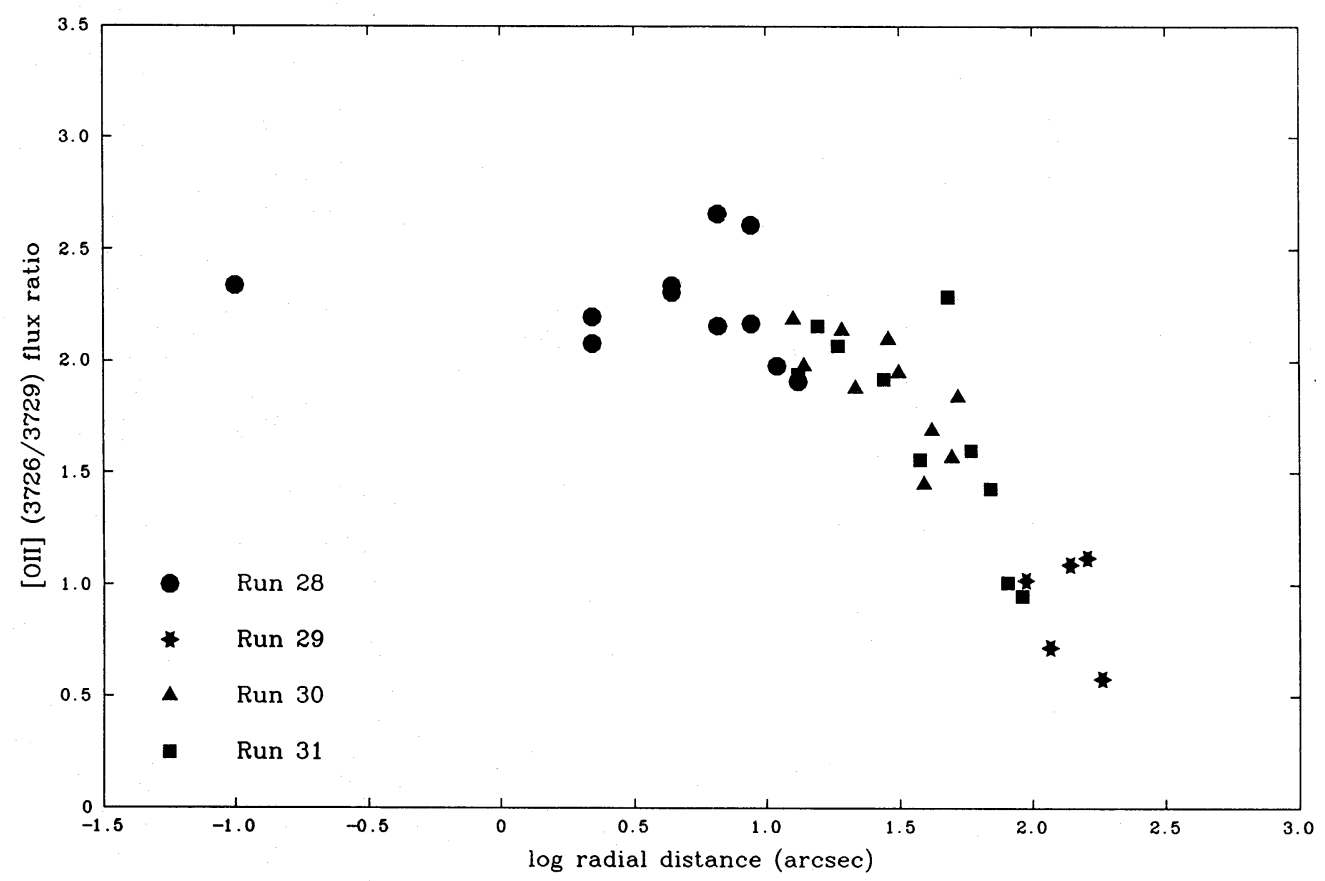

Figure 5. The solid symbols show the measured [O $\mathrm{II}]$ doublet flux ratios as a function of distance from the centre of IC 418 . An outwards decrease to values approaching the low-density limit can be seen.

arcsec. We have already shown in the previous section that there is a contribution to the extended [O $\mathrm{II}]$ emission from the seeing profile of the bright nebula. However, scattering cannot account for the observed fall in the $\left[\mathrm{O}_{\mathrm{II}}\right]$ doublet ratio with distance. In the previous section we also found that the observed [O II] surface brightness at large angular radii was significantly in excess of that predicted by the seeing profile (Fig. 3). We therefore hypothesize that the changing [O II] ratio in Fig. 5 is due to the superimposition upon the central [O II] seeing profile of relatively uniform extended emission from ionized material with an $[\mathrm{O} \mathrm{II}]$ doublet ratio near to the low-density limit.

We tested this interpretation by calculating the intensity distribution of the $\left[\mathrm{O}_{\mathrm{II}}\right]$ doublet from the central nebula due to seeing effects alone (assuming a constant doublet ratio of 2.25 ) and subtracting this from the observed [ $\left.\mathrm{O}_{\mathrm{II}}\right]$ profile at each spatial position. The residual of this procedure should give the $\left[\mathrm{O}_{\mathrm{II}}\right]$ flux and doublet ratio corrected for seeing effects. We found that, particularly in the inner regions $(<30$ arcsec), the corrected profiles tended to be dominated by the noise introduced by the subtraction procedure. A merger of all the residual $\left[\mathrm{O}_{\mathrm{II}}\right]$ profiles obtained at angular radii larger than 30 arcsec gave a $3726: 3729$ ratio of $1.00 \pm 0.36$, significantly lower than the central ratio of $2.25 \pm 0.02$ and consistent with the presence of extended low-density emission around IC 418.

\subsection{The low-density [O II] emission around IC 418}

As discussed in Sections 3.3 and 3.4, neither the brightness distribution nor the change in doublet ratio of the [O II] emission, at angular distances larger than 30 arcsec from the centre of IC 418, can be accounted for by the extended image seeing profile (Fig. 3) and we now consider other possible sources for this emission.
One possible origin for the $[\mathrm{O} \mathrm{II}]$ emission at these large angular distances is that the intrinsic nebular emission is scattered by dust particles in a surrounding halo. All scattering mechanisms can be ruled out, however, by the observed change in the $\left[\mathrm{O}_{\mathrm{II}}\right] 3726,29 \AA$ doublet ratio at large angular radii (Fig. 5). No scattering mechanism could show such differential effects over such a short wavelength range.

Another process capable of producing the excess [O II] emission could be that a large low-density halo (diameter $>400$ arcsec) surrounds IC 418, and is photoionized by the central star. However, as it is thought that IC 418 is a young, compact, optically thick nebula (e.g. Middlemass 1988), difficulties arise in explaining the escape of the ionizing photons required to excite such a halo. It is possible that IC 418 has a nebular geometry that is optically thick in some directions but optically thin in others, an example of a nebula with such geometry being NGC 3918 (Clegg et al. 1987).

The most probable source of the excess extended [O II] emission around IC 418 is diffuse galactic background emission. Extended emission in $\mathrm{H} \alpha$, [N $\left.\mathrm{N}_{\mathrm{II}}\right] 6548,6584 \AA$, [O III $5007 \AA$ and $\left[\mathrm{S}_{\text {II }}\right] 6717,6731 \AA$ has been observed in many individual directions (Reynolds, Roesler \& Scherb 1974; Ogden \& Reynolds 1985; Reynolds 1985a,b; Reynolds 1987). Most of these observations have been made fairly close to the galactic plane, but some have included regions at higher latitudes, and large-scale maps of diffuse background $\mathrm{H} \alpha$ emission are presented by Reynolds \& Ogden (1979). No direct observations of diffuse galactic background $\left[\mathrm{O}_{\mathrm{II}}\right]$ emission have yet been reported, so to predict an approximate [ $\left.\mathrm{O}_{\mathrm{II}}\right]$ strength for the region around IC 418 it was necessary to adopt a model for the spectrum of the background emission. As pointed out by Mathis (1986), the shock models of Raymond (1976) and Shull \& McKee (1979). can produce spectra similar to the observed diffuse background emission for shock speeds of $60-90 \mathrm{~km} \mathrm{~s}^{-1}$, 
although an expanding supernova remnant (SNR) would only spend $\sim 10$ per cent of its time at these shock speeds and so an explanation of the observed diffuse emission in the many lines of sight reported would require the SNR causing each shock to be at a very similar stage of development. Photoionization models of the diffuse background emission (Mathis 1986) have less fundamental problems, and reproduce the observed emission-line ratios under certain conditions. The main constraint on these models is that the photons that cause the ionization need to travel large distance (up to hundreds of parsecs) from the $\mathrm{O}$ stars that produce them to the regions of diffuse emission, which requires that 10 per cent of the sky, as seen from a typical $\mathrm{O}$ star, has a very low hydrogen column density, $<10^{17} \mathrm{~cm}^{-2}$, over such distances (Mathis 1986).

To determine the level of diffuse background [O II] emission, it is possible to adopt either the Mathis model for $q=-3$, or the Shull \& McKee model for a shock speed of 80 $\mathrm{km} \mathrm{s}^{-1}$. Both give good agreement with the observed $\mathrm{H} \alpha$, [O III], [N $\mathrm{NI}]$ and $[\mathrm{S} \mathrm{II}]$ emission, whilst predicting [O $\mathrm{OI}]$ $3726+3729 / \mathrm{H} \alpha$ ratios of 1.01 and 0.86 , respectively. Within the uncertainties in the models and the variation in the spectrum of the diffuse background emission with line-ofsight, these two predicted values are similar enough to adopt $\left[\mathrm{O}_{\mathrm{II}}\right] / \mathrm{H} \alpha=1.0$ as representative of the diffuse background emission.

IC 418 lies in a region of enhanced $\mathrm{H} \alpha$ emission, close to the Orion-Eridanus region, and, from the $\mathrm{H} \alpha$ contour map of the diffuse background emission in this region (Reynolds \& Ogden 1979), we estimate $20 \mathrm{R}<I(\mathrm{H} \alpha)_{\text {diff }}<40 \mathrm{R}$ for the general region around IC 418 , and $I(\mathrm{H} \alpha)_{\text {diff }}=20 \mathrm{R}$ at the coordinates of IC 418 [1 Rayleigh $(1 \mathrm{R})=5.64 \times 10^{-18} \mathrm{erg}$ $\mathrm{cm}^{-2} \mathrm{~s}^{-1} \operatorname{arcsec}^{-2}$ at the wavelength of $\mathrm{H} \alpha$ ]. We adopt [O II] $\mathrm{H} \alpha=1.0$, i.e. $I\left(\mathrm{O}_{\mathrm{II}}\right)_{\text {diff }} \sim 1.1 \times 10^{-16} \mathrm{erg} \mathrm{cm}^{-2} \mathrm{~s}^{-1} \operatorname{arcsec}^{-2}$. This level of diffuse background radiation is indicated by the dashed line in Fig. 3 and it could clearly account for the excess $[\mathrm{OI}]$ emission observed at angular distances of 30-200 arcsec from IC 418. Indeed, a combination of the extended seeing profile of IC 418 and the predicted diffuse galactic background emission can provide an almost exact fit to the observed data (dotted line in Fig. 3).

We therefore conclude that a combination of the extended nebular seeing profile plus diffuse galactic background emission provides the most probable explanation for the extended line emission seen around IC 418. We also conclude, from Figs 4 and 5, that the extended line emission observed by Baessgen et al. (1987), out to 40 arcsec from the centre of IC 418 , is likely to have been due to atmospheric scattering alone, rather than an ionized outflow.

\section{EXTENDED EMISSION AROUND THE SYMBIOTIC STAR RX PUPPIS}

\subsection{Line intensities and the seeing profile around RX Puppis}

The four 2D spectra of RX Pup (Runs 32-35; see Table 1) were taken with the same instrumental set-up as for IC 418 (Runs 28-31). Runs 33-35 were centred on RX Pup and will be referred to as the 'on-star' images. For Run 32 the slit was centred 14 arcsec north of RX Pup and this will be referred to as the 'off-star' image. Exposures with different ND filters were required for the 'on-star' spectra in order that both strong and weak lines be detected with a sufficient signal-tonoise ratio.

For each of the 'on-star' runs, the object was detected in spatial increments 23-30, and spectra were extracted by coadding every two contiguous spatial increments, to give four spectra in all. The flux in the strong emission lines of [ $\mathrm{Ne} \mathrm{v}]$ $3426 \AA$, [Ne III] $3868 \AA$, and $\mathrm{H} \delta$, together with the weak [O II $3726+3729 \AA$ doublet flux, was measured in each spectrum and the surface brightnesses in these lines from all three 'on-star' runs (neglecting saturated lines) are presented in Table 4 as a function of angular distance from the centre of RX Pup.

In the exposure positioned 14 arcsec north of RX Pup, [O II $3726+3729 \AA$ emission was detected across the full image and, to improve the signal-to-noise ratio, every five contiguous spatial increments were co-added to give 10 extracted spectra. Table 5 presents the observed surface brightness for [Ne v] $3426 \AA$, [O II] $3726+3729 \AA$, [Ne III] $3868 \AA$ and $\mathrm{H} \delta$, as a function of angular distance from RX Pup, where this distance is measured from the central spatial increment of the merged 'on-star' spectrum.

The most striking features in a comparison between Tables 4 and 5 are the increase in the relative strength of the [O II] $3726+3729 \AA$ lines in the 'off-star' position, and the uniform level of the [O II] $3726+3729 \AA$ flux at the 10 'offstar' positions. Fig. 6(a and b, respectively) presents the mean 'on-star' spectrum of RX Pup and the 'off-star' spectrum at the subtangent position 14 arcsec north of RX Pup (spatial increments 21-30). The change in the strength of $\left[\mathrm{O}_{\text {II }}\right] 3726+3729 \AA$, relative to [Ne v] $3426 \AA$, [Ne III] $3868 \AA$ and $\mathrm{H} \delta$, is striking. A comparison between the various line ratios as a function of angular distance is hindered by the low signal level of the $[\mathrm{Ne} \mathrm{v}] 3426 \AA$, [ $\mathrm{Ne}$ III] $3868 \AA$ and $\mathrm{H} \delta$ lines at radial distances $>15$ arcsec, and the weak contrast of $\left[\mathrm{O}_{\mathrm{II}}\right] \quad 3726+3729 \AA$ at the 'on-star' position. However, a comparison between the relative line strengths in the central increments of the 'on-star' position,

Table 4. Surface brightness in [Nev] $3426 \AA$, [O II] $3726+3729 \AA$, [Ne III] $3868 \AA$ and $\mathrm{H} \delta$ for RX Pup at the 'on-star' position.

\begin{tabular}{|c|c|c|c|c|c|}
\hline \multirow{2}{*}{$\begin{array}{c}\text { Spatial } \\
\text { Increment }\end{array}$} & \multirow{3}{*}{$\begin{array}{c}\text { Radial } \\
\text { Distance } \\
(\operatorname{arcsec})\end{array}$} & \multicolumn{4}{|c|}{ Surface brightness $\left(\times 10^{-15} \mathrm{ergs} \mathrm{cm}^{-2} \mathrm{sec}^{-1} \operatorname{arcsec}^{-2}\right)$} \\
\hline & & {$[\mathrm{Ne} \mathrm{V}]$} & [O II ] & [Ne III ] & $\mathrm{H} \delta$ \\
\hline & & $3426 \AA$ & $3726+3729 \AA$ & $3868 \AA$ & $4101 \AA$ \\
\hline $23+24$ & 6.6 & 9.5 & - & 9.6 & 2.7 \\
\hline $25+26$ & 2.2 & 385.7 & $\sim 6.0$ & 275.4 & 103.5 \\
\hline $27+28$ & 2.2 & 440.0 & $\sim 6.0$ & 309.9 & 95.8 \\
\hline $29+30$ & 6.6 & 8.6 & - & 6.2 & 3.7 \\
\hline
\end{tabular}


Table 5. Surface brightness in [Ne v] $3426 \AA$, [O II] $3726+3729 \AA$, [Ne III] $3868 \AA$ and $\mathrm{H} \delta$ for RX Pup at the 'off-star' position.

\begin{tabular}{|c|c|c|c|c|c|}
\hline \multirow{3}{*}{$\begin{array}{l}\text { Spatial } \\
\text { Increment }\end{array}$} & \multirow{3}{*}{$\begin{array}{c}\text { Radial } \\
\text { Distance } \\
\text { (arcsec) }\end{array}$} & \multicolumn{4}{|c|}{ Surface brightness $\left(\times 10^{-17} \mathrm{ergs} \mathrm{cm}^{-2} \sec ^{-1} \operatorname{arcsec}^{-2}\right)$} \\
\hline & & {$[\mathrm{NeV}]$} & [O II ] & [Ne III ] & $\mathrm{H} \delta$ \\
\hline & & $3426 \AA$ & $3726+3729 \AA$ & $3868 \AA$ & $4101 \AA$ \\
\hline $1-5$ & 53.6 & - & 12.2 & - & 1.8 \\
\hline $6-10$ & 43.0 & - & 11.9 & - & 1.4 \\
\hline $11-15$ & 32.8 & - & 12.3 & - & 0.7 \\
\hline $16-20$ & 23.4 & 6.2 & 9.6 & 1.8 & 1.2 \\
\hline 21-25 & 16.0 & 6.5 & 11.7 & 6.3 & 4.0 \\
\hline $26-30$ & 14.4 & 10.9 & 13.1 & 6.5 & 3.9 \\
\hline $31-35$ & 20.0 & 5.4 & 11.5 & 1.3 & 1.7 \\
\hline $36-40$ & 28.9 & - & 10.8 & - & 1.2 \\
\hline $41-45$ & 38.9 & - & 11.3 & - & - \\
\hline $46-50$ & 49.3 & - & 13.6 & - & - \\
\hline
\end{tabular}
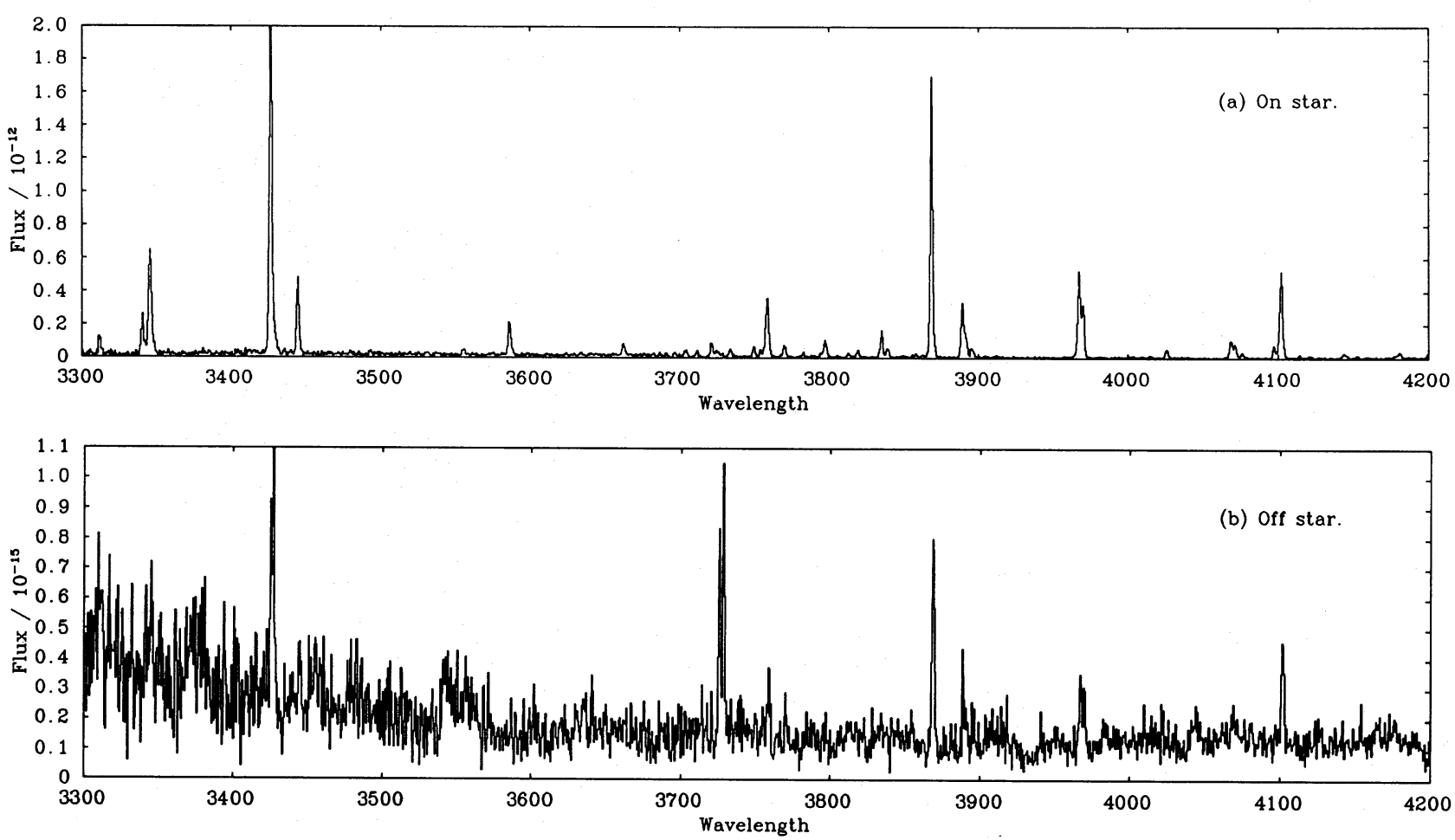

Figure 6. The 3300-4200 A spectrum of (a) RX Pup, and (b) a position centred 14 arcsec north of RX Pup. Note the very large increase in the strength of $\left[\mathrm{O}_{\text {II }}\right] 3726,3729 \AA$ relative to the other lines at the off-star position.

with those at the subtangent 'off-star' position, shows that at the 'off-star' position the $\left[\mathrm{O}_{\mathrm{II}}\right] \quad 3726+3729: \mathrm{H} \delta$ ratio increases by a factor of $\sim 13$ and the [O II] $3726+3729:[\mathrm{Ne}$ III] 3868 ratio by a factor of $\sim 20$, whilst the ratios involving emission lines other than [O II] $3726+3729 \AA$ remain the same within the errors. The 'onstar' to 'off-star' surface brightness ratio for [O II] $3726+3729 \AA$ is $\sim 200$, while those of [Nev] $3426 \AA$, [Ne III $3868 \AA$, and $\mathrm{H} \delta$, are $\sim 6000, \sim 4250$ and $\sim 2500$, respectively. Clearly, the [O II $] 3726+3729 \AA$ surface brightness at the 'off-star' positions is a factor of 10-25 too intense relative to the other line to be explained by scattering effects, atmospheric or otherwise.

As confirmation of this, Fig. $7(\mathrm{a}-\mathrm{d})$ presents the observed surface brightness distributions around RX Pup, as a func- tion of angular distance from the centre of the object, for $[\mathrm{Ne} \mathrm{v}] 3426 \AA,[\mathrm{Ne}$ III $] 3868 \AA, \mathrm{H} \delta$ and $\left[\mathrm{O}_{\mathrm{II}}\right] 3726+3729 \AA$, respectively, with the point source seeing profile (solid line) normalized to the observed surface brightness at 2.2 arcsec. This normalization is approximate but was necessary as the emission lines of RX Pup are known to vary in strength and no recent absolute fluxes were available. However, our normalization implies 'integrated' fluxes of $F(3868)=$ $2.2 \times 10^{-11} \mathrm{erg} \mathrm{cm}^{-2} \mathrm{~s}^{-1}$ and $F(\mathrm{H} \delta)=0.8 \times 10^{-11} \mathrm{erg} \mathrm{cm}^{-2}$ $\mathrm{s}^{-1}$. The most recent absolute fluxes available in the literature were acquired in 1983 by Whitelock et al. (1984), who found $F(3868)=1.3 \times 10^{-11} \mathrm{erg} \mathrm{cm}^{-2} \mathrm{~s}^{-1}$ and $F(\mathbf{H} \delta)=$ $1.0 \times 10^{-11} \mathrm{erg} \mathrm{cm}^{-2} \mathrm{~s}^{-1}$ [in Section 5.1 our relative line fluxes for RX Pup are compared further with those of Whitelock et al. (1984)]. 

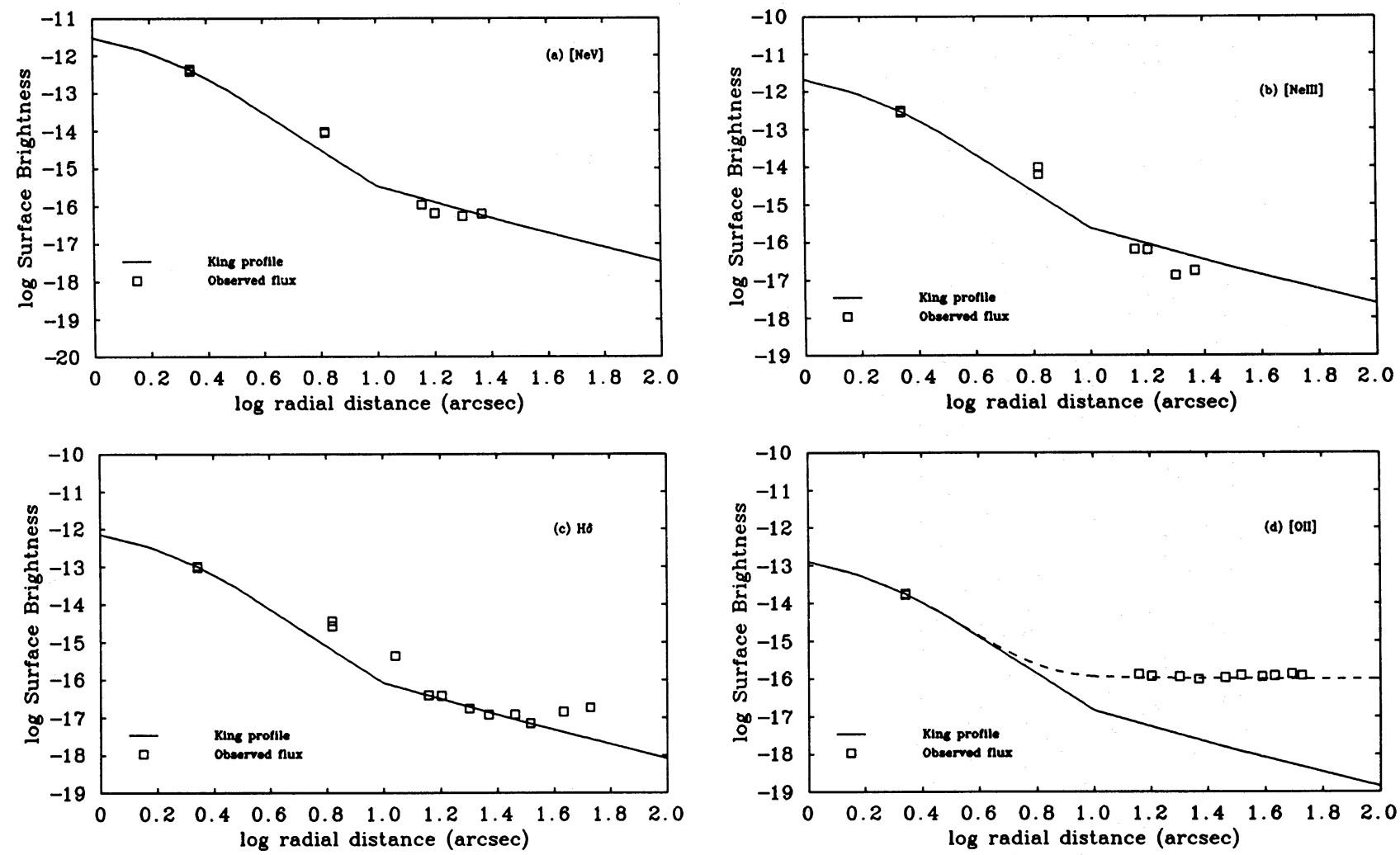

Figure 7. The solid lines show the predicted point source surface brightness seeing profiles (King profiles) for RX Pup, in the emission of (a)[Ne v] $3426 \AA,(\mathrm{b})\left[\mathrm{Ne}\right.$ III] $3868 \AA,(\mathrm{c}) \mathrm{H} \delta$ and (d) $\left[\mathrm{O}_{\mathrm{II}}\right] 3726,3729 \AA$.In each case the King profile has been normalized to the measured central intensities in the lines. The open symbols show the measured surface brightnesses (in units of erg cm $\mathrm{cm}^{-2} \mathrm{~s}^{-1} \mathrm{arcsec}^{-2}$ ) at various radial distances from the centre of RX Pup. In (d) the dashed line shows the predicted level of diffuse galactic emission in [O II] (see text).

Given the likely errors involved in this type of fit, there is good agreement between the adopted seeing profiles and the observed surface brightness distributions of $[\mathrm{Ne} \mathrm{v}] 3426 \AA$, [Ne III $3868 \AA$ and $\mathrm{H} \delta$, although there is some indication that there is excess flux in $\mathrm{H} \delta$ at angular distances $>40$ arcsec. In each of the fits to the observed [Ne v] $3426 \AA$, [Ne III] $3868 \AA$ and $\mathrm{H} \delta$ surface brightness distributions there is an indication that the seeing profile should be wider at distances of 6-10 arcsec, which is probably due to the fact that the FWHM of the inner seeing profile was broader at the time of our observations than in the model of King (1971).

The observed $\left[\mathrm{O}_{\mathrm{II}}\right] 3726+3729 \AA$ surface brightness distribution, however, shows poor agreement with the predicted profile, and appears to have a flat brightness distribution at angular distances $>15$ arcsec. Although the central object $\left[\mathrm{O}_{\mathrm{II}}\right]$ doublet is of low contrast, its flux would need to be a factor of 10 larger than that measured in order to account for the observed level of the extended [O II] $3726+3729 \AA$ emission. The change in the [O II] 3726:3729 $\AA$ doublet ratio (Section 4.2) and the large change in $\left[\mathrm{O}_{\mathrm{II}}\right] 3726+3729 \AA$, relative to the other lines, are also in agreement with the fact that seeing alone cannot account for the $\left[\mathrm{O}_{\text {II }}\right] 3726+3729 \AA$ flux beyond 15 arcsec.

\subsection{The [O II] doublet ratio around RX Puppis}

Fig. $8(\mathrm{a}$ and b) presents two spectra of the $[\mathrm{O}$ II] 3726, $3729 \AA$ spectral region: the first from a spectrum centred on
RX Pup (Run 33); and the second an 'off-star' spectrum from Run 32, obtained from an extraction of all spatial increments where the object was detected. There is an obvious difference between the $[\mathrm{O} \mathrm{II}]$ doublet ratio at each of these positions, with the 'on-star' [O $\left.\mathrm{O}_{\text {II }}\right]$ 3726:3729 $\AA$ ratio being $>2.0$ and the 'off-star' ratio being $<1.0$. Due to the poor signal-to-noise ratio, the $\left[\mathrm{O}_{\text {II }}\right]$ 3726:3729 $\AA$ ratio from the 'onstar' image was difficult to measure. However, using the emission line fitting routine ELF, we were able to estimate the [O II $] 3726: 3729 \AA$ ratio as $2.9 \pm 0.50$. For the 'off-star' image, 20 separate spectra, each of five contiguous spatial increments, were extracted, the Gaussian fit to the observed [O II] 3726:3729 A ratio being similar in each case. A merger of all 50 spatial increments give a higher signal-to-noise ratio and the ELF Gaussian line fit to the $[\mathrm{O}$ II $] 3726,3729$ A doublet yielded a ratio of $0.70 \pm 0.05$. The 'on-star' and 'off-star' [O II] 3726:3729 A ratios of 2.9 and 0.70 are near the high- and low-density limits, respectively, and imply electron densities of $>20000 \mathrm{~cm}^{-3}$ at the 'on-star' position and $<100 \mathrm{~cm}^{-3}$ at the 'off-star' position.

As there is a clear change in the $[\mathrm{O} I 1]$ doublet ratio between the 'on-star' and 'off-star' positions, this rules out scattering of the symbiotic star emission lines as the mechanism producing the observed extended [O II] 3726, $3729 \AA$ emission. The large change in the intensity of [O II] $3736+3729 \AA$ relative to the other emission lines, and its uniform brightness over the 15-50 arcsec range observed (Section 4.1), also indicate a non-scattering source. 

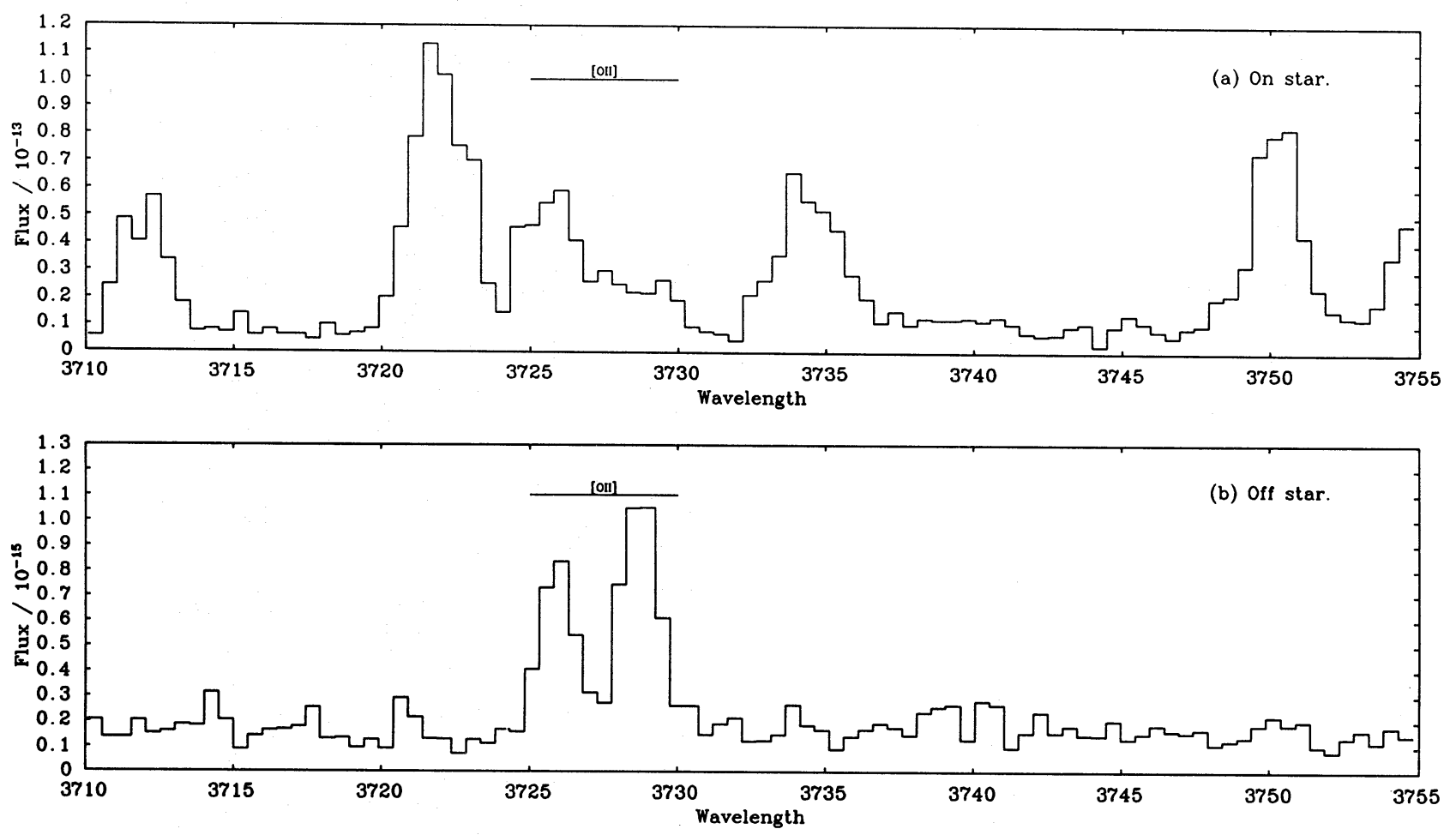

Figure 8. The $\left[\mathrm{O}_{\mathrm{II}}\right]$ doublet observed in the spectrum of (a) RX Pup and (b) a position 14 arcsec north of RX Pup. The $3726 / 3729$ intensity ratio is found to be at the high- and low-density limits in $(a)$ and $(b)$, respectively.

\subsection{The low-density [O II] emission around RX Puppis}

As with IC 418, the low-density [O II] 3726+3729 A emission, detected north of RX Pup, cannot be accounted for by the theoretical seeing profile, and is not due to scattering of the central $[\mathrm{OI}]$ emission. The source of the extended emission could be material associated with RX Pup, but, as was the case for IC 418, the star lies in a region of enhanced diffuse galactic background emission, in this case the Gum Nebula.

The wide-angle $\mathrm{H} \alpha$ photograph of this region, by Sivan (1974), shows bright emission from the Gum Nebula in the range $240^{\circ}<l<276^{\circ}$ and $+17^{\circ}<b<-19^{\circ}$. The position of $\operatorname{RX} \operatorname{Pup}\left(l=258.5^{\circ}, b=-3.9^{\circ}\right)$ is in the southern part of this region within a bright ring of $\mathrm{H} \alpha$ emission. A detection of the diffuse galactic background $\mathrm{H} \alpha$ in this region $\left(l=240^{\circ}\right.$, $\left.b=0.0^{\circ}\right)$ gives $I(\mathrm{H} \alpha)_{\text {diff }} \sim 1.0 \pm 0.3 \times 10^{-16} \mathrm{erg} \mathrm{cm}^{-2} \mathrm{~s}^{-1}$ $\operatorname{arcsec}^{-2}$ (Reynolds et al. 1974), whilst observations at eight discrete positions towards the Gum Nebula (Reynolds 1976) give a range of $\mathrm{H} \alpha$ emission from $\sim 3 \times 10^{-16}$ to $\sim 15 \times$ $10^{-16} \mathrm{erg} \mathrm{cm}^{-2} \mathrm{~s}^{-1} \operatorname{arcsec}^{-2}$ within the nebula. Although the variation in $\mathrm{H} \alpha$ surface brightness in the Gum Nebula does not allow a specific value to be associated with the position of RX Pup, it should lie between the limits given above.

The observed $[\mathrm{O}$ III] $: \mathrm{H} \alpha$ and $[\mathrm{N} \mathrm{H}]: \mathrm{H} \alpha$ ratios within the Gum Nebula are $\sim 0.02-0.03$ and $\sim 0.5$, respectively (Reynolds 1976), which are consistent with the two models discussed in Section 3.5 for the diffuse galactic emission, and so the adoption of $I(3726+3729) / I(\mathrm{H} \alpha)=1$ should again be valid.

The observed diffuse [O II] $3726+3729 \AA$ A emission, along with the adopted point source seeing profile, are shown in Fig. $7(\mathrm{~d})$, where the dashed line gives the lower limit to the predicted combined flux from the seeing profile plus diffuse galactic background emission. The observed constant [O II] $3726+3729 \AA$ surface brightness of $\sim 1.2 \times 10^{-16} \mathrm{erg} \mathrm{cm}^{-2}$ $\mathrm{s}^{-1} \operatorname{arcsec}^{-2}$, along with the assumption that $\left[\mathrm{O}_{\mathrm{II}}\right] / \mathrm{H} \alpha \sim 1$, implies that the extended [O II] emission can be accounted for by the diffuse galactic background alone, without any need for a Gum Nebula contribution. Similarly, the observed $\mathrm{H} \delta$ surface brightness of $\sim 1.2 \times 10^{-17} \mathrm{erg} \mathrm{\textrm {cm } ^ { - 2 }} \mathrm{s}^{-1}$ $\operatorname{arcsec}^{-2}$, at angular distances $>40$ arcsec, can also be accounted for by assuming $\left[\mathrm{O}_{\mathrm{II}}\right] / \mathrm{H} \alpha \sim 1$ for the diffuse galactic background, along with the Case $\mathrm{B}$ recombination theory ratio of $\mathrm{H} \alpha / \mathrm{H} \beta \sim 11.5$ (Brocklehurst 1971). This gives a predicted level of $\sim 1.0 \times 10^{-17} \mathrm{erg} \mathrm{cm}^{-2} \mathrm{~s}^{-1}$ $\operatorname{arcsec}^{-2}$ for $\mathrm{H} \beta$.

\section{THE CENTRAL OBJECT SPECTRA}

\subsection{RX Puppis}

Table 6 presents the observed relative line intensities between 3200 and $4200 \AA$, measured from our 'on-star' RX Pup spectra (Runs 33-35; Table 1). We have chosen to normalize our line intensities to $F(\mathbf{H} \delta)=15.6$, the value measured by Whitelock et al. (1984) on a scale where $F(\mathrm{H} \beta)=100$. Table 6 also lists the relative line intensities measured by Whitelock $e t$ al. over the wavelength range in common. A comparison shows good agreement between the Balmer line relative intensities as discussed in Section 4.1, the absolute $\mathrm{H} \delta$ flux implied by our 1987 December 31 data agrees with that measured by Whitelock et al. (1984) on 1983 May 5. However, the [Ne III] 3868 and $3967 \AA$ lines in our 1987 spectra are both 2.5 times stronger than measured by Whitelock et al.(1984)in 1983, as is also the case for the [Fe viI], O III blend at $3760 \AA$. On the other 
Table 6. Emission-line fluxes of RX Pup.

\begin{tabular}{|c|c|c|c|}
\hline \multirow[b]{2}{*}{$\begin{array}{l}\lambda \\
(\AA)\end{array}$} & \multirow[b]{2}{*}{ Ion } & \multicolumn{2}{|c|}{$F(\lambda)$} \\
\hline & & $\begin{array}{l}\text { IPCS Run } \\
33,34,35\end{array}$ & $\begin{array}{l}\text { Whitelock } \\
\text { et al. (1984) }\end{array}$ \\
\hline 3299 & OIII & 1.0 & \\
\hline 3312 & OIII & 4.4 & \\
\hline 3341 & OIII & 7.9 & \\
\hline 3343 & {$[\mathrm{NeV}]+[\mathrm{NeIII}]$} & 23.7 & \\
\hline 3380 & OIV & 0.5 & \\
\hline 3385 & $\mathrm{OIII}+\mathrm{OIV}$ & 0.3 & \\
\hline 3410 & OIV & 0.6 & \\
\hline 3415 & OIII & 0.6 & \\
\hline 3426 & {$[\mathrm{NeV}]+\mathrm{O} I I I$} & 67.1 & \\
\hline 3444 & OIII & 12.0 & \\
\hline 3479 & $\mathrm{NIV}+\mathrm{HeI}$ & 0.2 & \\
\hline 3554 & $\mathrm{HeI}$ & 1.0 & \\
\hline 3587 & {$[\mathrm{Fe} \mathrm{VII}]+\mathrm{HeI}$} & 6.5 & \\
\hline 3635 & $\mathrm{HeI}$ & 0.4 & \\
\hline 3692 & H18 & 0.6 & \\
\hline 3697 & H17 & 0.9 & \\
\hline 3704 & $\mathrm{H} 16+\mathrm{He} \mathrm{I}+\mathrm{O} I I I$ & 1.6 & \\
\hline 3712 & H15 & 1.2 & \\
\hline 3722 & $\mathrm{H} 14+[\mathrm{S} \mathrm{III}]$ & 2.7 & \\
\hline 3727 & {$[\mathrm{OII}]$} & 2.1 & \\
\hline 3734 & $\mathrm{H} 13$ & 1.8 & \\
\hline 3750 & $\mathrm{H} 12$ & 2.0 & \\
\hline 3754 & OIII & 0.8 & \\
\hline 3760 & {$[\mathrm{Fe} \mathrm{VII}]+\mathrm{OIII}$} & 13.6 & 5.5 \\
\hline 3771 & H11 & 2.4 & - \\
\hline 3783 & HeII & 0.9 & - \\
\hline 3798 & $\mathrm{H} 10$ & 3.9 & 4.2 \\
\hline 3814 & $\mathrm{HeI}$ & 0.8 & - \\
\hline 3820 & $\mathrm{HeI}$ & 1.2 & - \\
\hline 3835 & H9 & 4.3 & 4.3 \\
\hline 3839 & {$[\mathrm{FeV}]$} & 1.7 & - \\
\hline 3854 & Si II & 0.2 & - \\
\hline 3858 & HeII & 0.4 & - \\
\hline 3863 & Si II & 0.4 & - \\
\hline 3868 & [NeIII] & 42.4 & 18.6 \\
\hline 3889 & $\mathrm{H} 8+\mathrm{HeI}$ & 13.3 & 8.7 \\
\hline 3896 & {$[\mathrm{FeV}]$} & 1.8 & - \\
\hline 3924 & HeII & 0.2 & - \\
\hline $\begin{array}{l}3967 \\
3970\end{array}$ & [NeIII] & 17.2 & 13.3 \\
\hline $\begin{array}{l}3970 \\
4026\end{array}$ & $\begin{array}{l}\mathrm{H} 7 \\
\mathrm{HeI}+\mathrm{HeII}\end{array}$ & $\begin{array}{l}6.0 \\
1.5\end{array}$ & $\int_{1.5}$ \\
\hline 4068 & {$[\mathrm{SII}]$} & 3.3 & 4.6 \\
\hline 4071 & {$[\mathrm{FeV}]$} & 1.8 & \\
\hline 4076 & [S II ] & 0.9 & - \\
\hline 4096 & N III & 2.1 & - \\
\hline 4101 & $\mathrm{H} \delta$ & 15.6 & 15.6 \\
\hline 4121 & $\mathrm{HeI}+\mathrm{OIII}$ & 0.5 & - \\
\hline 4144 & $\mathrm{HeI}$ & 1.0 & - \\
\hline 4181 & {$[\mathrm{FeV}]$} & 1.6 & - \\
\hline 4200 & HeII & 0.7 & - \\
\hline 4861 & $\mathrm{H} \beta$ & - & 100.0 \\
\hline
\end{tabular}

hand, the 1984 April spectrum of RX Pup, illustrated in Allen \& Wright (1988), appears to show [Ne III] to Balmer line ratios very similar to those measured in our 1987 spectra. It therefore seems that the evolution of the spectrum of RX Pup towards higher excitation, that began in 1979 (Klutz \& Swings 1981), stabilized between 1983 and 1984.

\subsection{IC 418}

Table 7 presents the relative line intensities measured from our central object spectra of IC 418. These intensities have been dereddened by $c(\mathbf{H} \beta)=0.30$, using the reddening law of Howarth (1983), and normalized as described in the
Table 7. Emission-line fluxes of IC 418 dereddened using $c(\mathrm{H} \beta)=0.30$, relative to $\mathrm{H} \beta=100$ (TPP), $\mathrm{H} \alpha=285.0$ (IPCS Runs 116 and 118 ), $\mathrm{H} \gamma=46.9$ (IPCS Runs 155, 156 and 159), or $\mathrm{H} \delta=26.0$ (IPCS Run 28).

\begin{tabular}{|c|c|c|c|c|c|}
\hline $\begin{array}{c}\lambda \\
(\AA)\end{array}$ & Ion & TrP & $\begin{array}{c}I(\lambda) \\
\text { IrCS Run } \\
28\end{array}$ & $\begin{array}{l}\text { IPCS Runs } \\
155,156,159\end{array}$ & $\begin{array}{c}\text { IrCS Runs } \\
116,118\end{array}$ \\
\hline 3712 & $\mathrm{H} 15$ & - & - & 1.8 & \\
\hline 3722 & $\mathrm{H} 14+[\mathrm{S} \mathrm{III}]$ & - & 2.9 & 2.9 & \\
\hline 3726 & {$[\mathrm{OII}]$} & 162.0 & 111.9 & 126.3 & \\
\hline 3729 & [OII] & & 49.8 & 59.9 & \\
\hline 3734 & H13 & - & 2.7 & 2.5 & \\
\hline 3750 & H12 & - & 2.9 & 3.1 & \\
\hline 3771 & H11 & - & 3.5 & 3.7 & \\
\hline 3798 & H10 & - & 4.8 & 4.4 & \\
\hline 3820 & $\mathrm{HeI}$ & - & 1.3 & 1.2 & \\
\hline 3835 & H9 & - & 6.5 & 6.6 & \\
\hline 3869 & [Ne III] & 2.8 & 3.4 & 5.1 & \\
\hline 3889 & $\mathrm{H} 8+\mathrm{HeI}$ & - & 14.9 & 15.0 & \\
\hline 3967 & [NeIII] & - & 0.7 & 0.5 & \\
\hline 3970 & H7 & - & 15.7 & 14.7 & \\
\hline 4026 & HeI & - & 1.8 & 1.3 & \\
\hline 4068 & {$[\mathrm{SII}]$} & 2.9 & 2.4 & 2.2 & \\
\hline 4076 & [SII] & & - & 1.0 & \\
\hline 4101 & $\mathrm{H} \delta$ & 25.7 & 26.0 & 26.5 & \\
\hline 4267 & $\mathrm{CII}$ & $\therefore$ & & 0.76 & \\
\hline 4340 & $\mathrm{H}_{\gamma}$ & 45.7 & & 46.9 & \\
\hline 4363 & [O III] & 0.9 & & 0.94 & \\
\hline 4388 & HeI & - & & 0.43 & \\
\hline 4472 & HeI & 4.0 & & 4.1 & \\
\hline 4861 & $\mathbf{H} \beta$ & 100.0 & & & \\
\hline 5007 & [O III] & 170.0 & & & \\
\hline 6548 & {$[\mathrm{~N} \mathrm{II}]$} & - & & & 44.1 \\
\hline 6564 & $\mathrm{H} \alpha$ & 281.8 & & & 285.0 \\
\hline 6578 & CII & - & & & 1.05 \\
\hline 6584 & [N II] & 147.9 & & & 148.0 \\
\hline 6622 & CII & - & & & $<0.32$ \\
\hline 6678 & $\mathrm{HeI}$ & 3.0 & & & 3.8 \\
\hline 6717 & {$[\mathrm{SII}]$} & 5.9 & & & 2.0 \\
\hline 6731 & {$[\mathrm{SII}]$} & & & & 4.0 \\
\hline 7065 & He I & 5.4 & & & 4.0 \\
\hline 7136 & [ArIII] & 6.6 & & & 4.7 \\
\hline 7236 & CII & - & & & 0.68 \\
\hline 7281 & He I & - & & & 0.66 \\
\hline 7325 & [OII] & 20.0 & & & 12.6 \\
\hline
\end{tabular}

Table 8. Quantal rates $(\dagger)$ for $C_{\text {II }}$ lines in IC 418.

$\begin{array}{cccc}\lambda & \begin{array}{c}\text { Predicted( } \ddagger) \\ \text { Case A }\end{array} & \begin{array}{c}\text { Predicted( } \ddagger) \\ \text { Case B }\end{array} & \text { Observed } \\ 4267 & & & \\ 7230 & 1.0 & 1.0 & 1.0 \\ 6578 & 0.061 & 3.2 & 1.5 \\ & 0.16 & 1.0 & 2.1\end{array}$

†Rate is $q(\lambda)=(\lambda / 4267)^{*}(F \lambda / F 4267)$.

$\ddagger$ From Pengelly (1963).

caption to Table 7. A comparison with the relative line intensities measured by Torres-Peimbert \& Peimbert (1977: TPP) shows good agreement, except for [O II $] 7325 \AA$ (see below).

We measured the fluxes of the $C_{\text {II }}$ lines 4267,6578 and $7236 \AA$ in IC 418 , and searched for the $4 f^{2} F^{0}-6 d^{2} D$ transition at $6622 \AA$, in order to study the recombination and fluorescence process in $\mathrm{C}_{\mathrm{II}}$ (Clegg et al. in preparation), as this nebula appears to have a '4267 $\AA$ problem' (Harrington et al. 1980; Torres-Peimbert, Peimbert \& Daltabuit 1980).

For the recombination line analysis we follow the methods of Clegg et al. (1983) and compute the quantal rate $q(\lambda)$ defined by them. In Table 8 , the observational data from Table 7 are compared with the calculations of Pengelly (1963), with $q$ given relative to the quantal rate in $\lambda 4267$. The $3 p-3 d 7236 \AA$ rate is sensitive to the assumption of Case A or B: when detailed balance occurs in the $2 p-3 d$ 
$687 \AA$ line, then the cascade proceeds via $\lambda \lambda 7231,7236$ rather than via $\lambda 687$. The measured $7236 \AA$ rate is close to the Case B value in IC 418; a similar result was found by Clegg et al. for NGC 40. The small departure from Case B is presumed to be due to escape of $687 \AA$ photons in the line wings. Pengelly did not include two-electron transitions in his calculations, but the radiative decay of $3 p^{2} P^{0}$ is actually dominated by such. We took Pengelly's effective recombination coefficient for the $3 p^{2} P^{0}$ level and calculated the fraction of decays proceeding via the observed line $\mathrm{C}_{\mathrm{II}}$ $3 s^{2} S-3 p^{2} P^{0}(J=3 / 2)$ at $6578 \AA$. With the Einstein A-values from Nussbaumer \& Storey (1981), and an assumed 2:1 ratio for the strengths of the two components of the $3 s-3 p$ multiplet, a fraction 0.20 of decays from $3 p$ proceed via $\lambda 6578$. (The other line component, $\lambda 6583$, is blended with [N II $] 6584 \AA$ ).

Table 8 shows that the $6578 \AA$ quantal rate in IC 418 is significantly larger than that calculated by Pengelly for either Case A or B. The reason is unknown. A new, more complete recombination calculation, including many two-electron transitions, would be useful to help study this problem.

It is also of interest to consider the mean electron densities that can be derived from the $\left[\mathrm{S}_{\mathrm{II}}\right]$ and $\left[\mathrm{O}_{\mathrm{II}}\right]$ doublet ratios in our on-object spectra of IC 418. In order to do this, we have adopted an electron temperature of $T_{\mathrm{c}}=8700 \mathrm{~K}$, the mean of the $\left[\mathrm{N}_{\text {II }}\right.$ ] electron temperatures of $8600 \mathrm{~K}$ and $8800 \mathrm{~K}$ that are obtained from the data of Aller \& Czyzak (1983) and TPP, respectively, for $c(\mathbf{H} \beta)=0.30$. After summing all spatial increments along the slit in our 1986 November red spectra, we find $I(6717) / I(6731)=0.488 \pm 0.018$. Using the same atomic data as Monk, Barlow \& Clegg (1988), we find that this ratio implies $n_{\mathrm{e}}\left(\mathrm{S}_{\mathrm{II}}\right)=11500 \pm 4000 \mathrm{~cm}^{-3}$.

The addition of all spatial increments along the slit across IC 418 in our 1986 November blue spectra gave $I(3726)$ / $I(3729)=2.11 \pm 0.04$, whilst our 1987 December spectrum (Run 28) gave a ratio of $2.25 \pm 0.02$. Finally, a spectrum of IC 418 obtained for wavelength calibration purposes on 1989 January 8 yielded a mean $\left[\mathrm{O}_{\mathrm{II}}\right]$ ratio of $2.07 \pm 0.08$. The weighted mean ratio obtained from these three measurements is thus $2.21 \pm 0.02$ which, for $T_{\mathrm{e}}=8700 \mathrm{~K}$, yields $n_{\mathrm{e}}\left(\mathrm{O}_{\mathrm{II}}\right)=6350 \pm 270 \mathrm{~cm}^{-3}$. For $T_{\mathrm{e}}=8700 \mathrm{~K}$, the $\left[\mathrm{O}_{\mathrm{II}}\right]$ $I(3727) / I(7325)$ ratio of 12.8 given by our spectra yields $n_{\mathrm{e}}=6460 \mathrm{~cm}^{-3}$, in good agreement with the density derived from the mean [O II] 3726:3729 ratio.

Finally, an estimate of the ionized nebular mass of IC 418 can be derived using the [O $\mathrm{OI}]$ electron density appropriate for the entire nebula. To do this, the dereddened $\mathrm{H} \beta$ flux and the nebular distance are also required. From the mean of values that appear in the literature, we have adopted $\log F(\mathbf{H} \beta)=-9.57 \pm 0.01$, along with $c(\mathrm{H} \beta)=0.30 \pm 0.04$. The Magellanic Cloud calibration for optically thick PN (Barlow 1987): $\log F(\mathrm{H} \beta)=-9.0 \pm 0.1$ at $1 \mathrm{kpc}$, then implies a distance of $1.4 \mathrm{kpc}$. However, IC 418 is a low-excitation nebula ([O III] $5007 \AA / \mathrm{H} \beta=1.7$ ) whose central star has probably not yet attained its plateau ionizing luminosity, and so the distance is likely to be overestimated by this method. The distance of $1 \mathrm{kpc}$, adopted by Taylor et al. (1989), seems reasonable and is adopted here also. To estimate the mass of ionized material in IC 418, an [O II] electron density appropriate for the entire nebula is required, whereas the values discussed above were for a 1.3-arcsec wide strip across this 12.4-arcsec diameter nebula. By assuming azimuthal symmetry for the nebula, we have used the [O II $] 3726,29 \AA$ surface brightnesses and doublet ratios given in Table 2 as a function of the radius (Run 28) in order to estimate the integrated nebular fluxes in [O II] 3726 and $3729 \AA$ (the values obtained from the eastern and western halves were averaged). An integrated $3726 / 3729$ flux ratio of 2.35 is the result, which corresponds to $n_{\mathrm{e}}=8740 \mathrm{~cm}^{-3}$ for $T_{\mathrm{e}}=8700 \mathrm{~K}$. Using these parameters in equation (5) of Barlow (1987), we derive an ionized hydrogen mass of 0.044 $M_{\odot}$ for IC 418 , which can be compared with the atomic hydrogen mass of $0.35 M_{\odot}$ derived by Taylor et al. (1989) for the circumnebular neutral shell.

\section{CONCLUSIONS}

In Sections 3 and 4 we concluded that our observations of extended line emission around both the planetary nebula IC 418 and the symbiotic star RX Pup can be matched by a combination of the effects of atmospheric scattering of the core object spectrum (close-in) plus diffuse galactic line emission (further out). Thus, in spite of the fact that planetary nebulae are often strong sources of IR dust emission, and that many are optically thick to ionizing radiation, so that a significant fraction of their dust masses may be expected to reside in circumnebular neutral zones, significant dustscattered line or continuum radiation has only so far been detected from NGC 7027 (Atherton et al. 1979; Middlemass, Clegg \& Walsh 1989). Middlemass et al. have presented quantitative models for reflection nebulae around planetary nebulae and discussed the difficulty of detecting these in the presence of scattering in the atmosphere and telescope. It would appear that spaceborne observations are more promising for the detection of dust-scattering effects, as atmospheric scattering will be eliminated, although instrumental scattering and diffuse galactic emission must still be contended with. Very careful ground-based spectropolarimetry at high signal-to-noise ratios may also be able to distinguish the effects of circumnebular dust scattering (see e.g. Leroy, Le Borgne \& Arnaud 1986). As discussed by Middlemass et al. (1989) for several other planetary nebulae, the effects of scattered light and diffuse galactic emission need to be allowed for to establish the presence of faint haloes around planetary nebulae. For the case of IC 418, we have concluded (Section 3.5) that a combination of the extended nebular seeing profile, plus diffuse galactic background emission, provides the most probable explanation for the extended emission observed by us. We also conclude from Figs 3 and 4 that the extended-line emission observed by Baessgen et al. (1987), out to 25 arscec from the centre of IC 418 (one of 11 bright PN for which faint haloes were claimed to have been detected by them), is likely to have been due to atmospheric scattering alone, rather than an extended ionized outflow.

\section{REFERENCES}

Allen, D. A. \& Wright, A. E., 1988. Mon. Not. R. astr. Soc., 232, 683. Aller, L. H. \& Czyzak, S. J., 1983. Astrophys. J. Suppl., 51, 211.

Atherton, P.D., Hicks, T. R., Reay, N. K., Robinson, G. J., Worswick, S. P. \& Phillips, J. P., 1979. Astrophys. J., 232, 789.

Baessgen, M., Cerrato, S. \& Grewing, M., 1987. Mitt. astr. Ges., Nr. 70, 347 . 
Barlow, M. J., 1987. Mon. Not. R. astr. Soc., 227, 161.

Brocklehurst, M., 1971. Mon. Not. R. astr. Soc., 153, 471.

Clavel, J. \& Flower, D. R., 1980. Mon. Not. R. astr. Soc., 190, 1 P.

Clegg, R. E. S., Harrington, J. P., Barlow, M. J. \& Walsh, J. R., 1987. Astrophys. J., 314, 551.

Clegg, R.E.S., Seaton, M.J.,Peimbert,M.\& Torres-Peimbert,S., 1983. Mon. Not. R. astr. Soc., 205, 417.

Cohen, M. \& Barlow, M. J., 1974. Astrophys. J., 193, 401.

Diego, F., 1985. Publs astr. Soc. Pacif., 97, 1209.

Harrington, J. P., Lutz, J. H., Seaton, M. J. \& Stickland, D. J., 1980. Mon. Not. R. astr. Soc., 191, 13.

Hollis, J. M., Oliverson, R. J., Kafatos, M. \& Michalitsianos, A. G., 1986. Astrophys. J., 301, 877.

Howarth, I. D., 1983. Mon. Not. R. astr. Soc., 203, 301.

Howarth, I. D. \& Murray, J., 1987. Starlink User Note, No. 50.

King, I. R., 1971. Publs astr. Soc. Pacif., 83, 199.

Klutz, M. \& Swings, J. P., 1981. Astr. Astrophys., 96, 406.

Knapp, G. R., 1986. Astrophys. J., 311, 731.

Knapp, G. R., Phillips, T. G., Leighton, R. B., Lo, K. Y., Wannier, P. G., Wootten, H. A. \& Huggins, P. J., 1982. Astrophys. J., 252, 616.

Leroy, J. L., Le Borgne, J. F. \& Arnaud, J., 1986. Astr. Astrophys., $160,171$.

Louise, R., Macron, A., Pascoli, G. \& Maurice, E., 1987. Astr. Astrophys. Suppl., 70, 201.

Mathis, J. S., 1986. Astrophys. J., 301, 423.

Mendez, R. H., Kudritzki, R. P., Herrero, A., Husfield, D. \& Groth, H. G., 1988. Astr. Astrophys., 190, 113.

Middlemass, D., 1988. Mon. Not. R. astr. Soc., 231, 1025.

Middlemass, D., Clegg, R. E. S. \& Walsh, J. R., 1989. Mon. Not. R. astr. Soc., 239, 5P.

Monk, D. J., Barlow, M. J. \& Clegg, R. E. S., 1988. Mon. Not. R. astr. Soc., 234, 583.

Monk, D. J., Barlow, M. J. \& Clegg, R. E. S., 1989. Planetary Nebulae, IAU Symp. No. 131, p. 197, ed. Torres-Peimbert, S.,
Kluwer, Dordrecht.

Mufson, S. L., Lyon, J. \& Marionni, P. A., 1975. Astrophys. J., 201, L85.

Nussbaumer, H. \& Storey, P. J., 1981. Astr. Astrophys., 96, 91.

Ogden, P. M. \& Reynolds, R. J., 1985. Astrophys. J., 290, 238.

Oke, J. B., 1974. Astrophys. J. Suppl., 27, 21.

Pengelly, R. M., 1963. PhD thesis, University of London.

Phillips, J. P., Sanchez Magro, C. \& Martinez Roger, C., 1984. Astr. Astrophys., 133, 395.

Phillips, J. P., Mampaso, A., Vilchez, J. M. \& Gomez, P., 1986. Astrophys. Space Sci., 122, 81.

Raymond, J., 1976. PhD thesis, University of Wisconsin.

Reynolds, R. J., 1976. Astrophys. J., 203, 151.

Reynolds, R. J., 1985a. Astrophys. J., 294, 256.

Reynolds, R. J., 1985b. Astrophys. J., 298, L27.

Reynolds, R. J., 1987. Astrophys. J., 323, 118.

Reynolds, R. J. \& Ogden, P. M., 1979. Astrophys. J., 229, 942.

Reynolds, R. J., Roesler, F. L. \& Scherb, F., 1974. Astrophys. J., 192, L53.

Shortridge, K., 1988. Starlink User Note, No. 86.

Shull, J. M. \& McKee, C. F., 1979. Astrophys. J., 227, 131.

Sivan, J. P., 1974. Astr. Astrophys. Suppl., 16, 163.

Storey, J. W. V., 1984. Mon. Not. R. astr. Soc., 206, 521.

Taylor, A. R. \& Pottasch, S. R., 1987. Astr. Astrophys., 176, L5.

Taylor, A. R., Gussie, G. T. \& Goss, W. M., 1989. Astrophys. J., 340, 932.

Torres-Peimbert, S. \& Peimbert, M., 1977. Rev. Mex. Astr. Astrofis., 2, 181.

Torres-Peimbert, S., Peimbert, M.\& Daltabuit, E., 1980. Astrophys. J., 238, 133.

Whitelock, P. A., Menzies, J. W., Lloyd Evans, T. \& Kilkenny, D., 1984. Mon. Not. R. astr. Soc., 208, 161.

Willner, S. P., Becklin, E. E. \& Visvanathan, N., 1972. Astrophys. J., $175,699$. 